\title{
Holographic complexity and de Sitter space
}

\author{
Shira Chapman, ${ }^{a}$ Damián A. Galante ${ }^{b}$ and Eric David Kramer ${ }^{c}$ \\ ${ }^{a}$ Department of Physics, Ben-Gurion University of the Negev, \\ Ben Gurion Blvd, Beer Sheva 84105, Israel \\ ${ }^{b}$ Department of Mathematics, King's College London, \\ the Strand, London WC2R 2LS, U.K. \\ ${ }^{c}$ Racah Institute of Physics, Hebrew University of Jerusalem, \\ Sderot Magnes, Jerusalem 91904, Israel \\ E-mail: schapman@bgu.ac.il, damian.galante@kcl.ac.uk, \\ ericdavidkramer@gmail.com
}

ABSTRACT: We compute the length of spacelike geodesics anchored at opposite sides of certain double-sided flow geometries in two dimensions. These geometries are asymptotically anti-de Sitter but they admit either a de Sitter or a black hole event horizon in the interior. While in the geometries with black hole horizons, the geodesic length always exhibit linear growth at late times, in the flow geometries with de Sitter horizons, geodesics with finite length only exist for short times of the order of the inverse temperature and they do not exhibit linear growth. We comment on the implications of these results towards understanding the holographic proposal for quantum complexity and the holographic nature of the de Sitter horizon.

Keywords: 2D Gravity, AdS-CFT Correspondence

ArXiv EPrint: 2110.05522 


\section{Contents}

1 Introduction 1

2 2d dilaton-gravity theories 5

$\begin{array}{lll}2.1 & \text { Penrose diagram coordinates } & 6\end{array}$

3 Geodesics in 2d spacetimes $\quad 7$

3.1 Geodesics for general $f(r)$ geometries $\quad 7$

3.2 Geodesics are always maximal $\quad 9$

$\begin{array}{ll}3.3 \text { Working in dimensionless coordinates } & 10\end{array}$

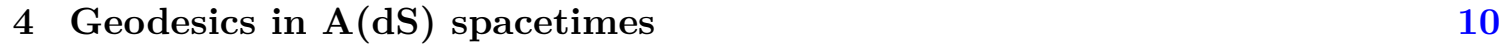

$\begin{array}{lll}4.1 & \text { Geodesics in global } \mathrm{AdS}_{2} & 10\end{array}$

$\begin{array}{lll}4.2 & \text { Geodesics in the } \mathrm{AdS}_{2} \text { black hole } & 12\end{array}$

$\begin{array}{lll}4.3 \text { Geodesics in } \mathrm{dS}_{2} & 14\end{array}$

5 Geodesics in flow geometries $\quad \mathbf{1 5}$

$\begin{array}{lll}5.1 & \text { Geodesics in the centaur geometry } & 15\end{array}$

$\begin{array}{lll}5.2 \text { Geodesics in } \gamma \text {-centaurs } & 18\end{array}$

5.3 Geodesics in AdS-to-AdS geometries 23

6 Discussion $\quad 25$

A Thermodynamics of dilaton-gravity theories and $\gamma$-centaur geometries 29

B From conformal coordinates to the Schwarzschild gauge 30

C Exact volume and boundary time in $\gamma$-centaur geometries 31

\section{Introduction}

The objective of this paper is to study cosmological event horizons from a modern holographic perspective. Cosmological horizons surround observers in universes with a positive cosmological constant, like the one our own universe asymptotes to. Despite their obvious relevance, they have been much less explored compared to black hole horizons. Cosmological horizons behave differently from black hole horizons, see e.g., [1, 2], even though, for instance, their entropy is also proportional to the area of the event horizon [3]. 
The AdS/CFT correspondence [4] provides a fruitful framework for studying black hole horizons in negatively curved spaces using conformal field theory (CFT) observables located at the boundary. In this arena, a special role is played by tools from the world of quantum information, see e.g., [5-7]. It would be surprising if similar tools did not play a central role in understanding the cosmological horizon as well. However, the fact that there is no timelike boundary in de Sitter spacetime $(\mathrm{dS})$ is an obstacle in translating ideas from gravitational holography into the cosmological case.

Past attempts to study de Sitter holographically include the dS/CFT correspondence [8-13], thinking of the dS horizon as a holographic screen, e.g., [14-16] and static patch holography which associates a quantum mechanical model with the observer's worldline $[17,18]$. Recently, a new set of ideas appeared including the use of $T \bar{T}$ deformations [19-21], a cosmological bootstrap program [22-28], and studies of the Euclidean partition function in 2d quantum gravity [29-31]. It is fair to say, though, that still there is no single microscopic quantum model to describe the cosmological horizon.

Interest in a holographic description of $\mathrm{dS}$ is further motivated by the fact that it has been alarmingly difficult to find stable de Sitter-like vacua in String Theory. The few successful attempts such as [32] are still a matter of controversy. Some authors have gone so far as to conjecture that there do not exist stable de Sitter-like vacua in String Theory [33]. While it is indeed important to continue the search for stable vacua, further study of holography, especially the question of whether a holographic description of dS is even possible, is an alternative way of exploring quantum gravity in dS.

Here, we continue the effort started in $[34,35]$ to probe the cosmological horizon using the standard tools of the AdS/CFT correspondence. The main idea behind this program is to embed part of a dS universe inside AdS and, in doing so, providing a boundary to study the cosmological horizon. Embedding $\mathrm{dS}_{d+1}$ inside $\mathrm{AdS}_{d+1}$ in $d>1$ was first attempted in $[36,37]$, where it was observed that in order to satisfy the null energy condition, it was necessary to hide the dS patch inside a black hole horizon in AdS. However, a new set of geometries was proposed in $[34,35]$, where the cosmological event horizon is in causal contact with the AdS boundary. These are solutions to certain dilaton-gravity theories in two dimensions that, when uplifted to higher dimensions, do satisfy the null energy conditions [38]. They appear in the context of the recently studied near $\mathrm{AdS}_{2}$ geometries [39-42], where there is a large, slowly varying dilaton playing the role of the size of the compact dimensions. These geometries share similar features to the low energy regime of the SYK model $[43,44]$. From this point of view, they can be seen as an RG flow from a UV near-conformal point towards a dS infrared point and we therefore refer to them as flow geometries. One could imagine building the dual to the flow geometries from relevant deformations of SYK-like models [38]. ${ }^{1}$

From a macroscopic perspective, the flow geometries allow us to compute different types of observables in the hope of characterising the cosmological event horizon. These observables turn out to differ significantly from their counterparts for black hole horizons.

\footnotetext{
${ }^{1}$ It was conjectured in [38] that the coupling of the relevant deformation might need to be complexified in order for the bulk to exhibit a flow towards de Sitter.
} 
Examples of these include: the frequencies of the dissipative quasinormal modes, which have a small real part which indicates that the geometry in the deconfined phase is less efficient at thermalizing [34]; the out-of-time-ordered four point function, that oscillates in time rather than obeying the acclaimed exponential growth of chaotic systems [35]; and, positive energy shockwaves, which open the wormhole rather than closing it [45]. All these pose challenges in the microscopic interpretation of the flow geometries.

Furthermore, in three dimensions, corrections to the cosmological horizon entropy were recently computed, finding again notable differences with respect to analogous corrections for black holes $[46,47]$.

In this paper, we concentrate on a different macroscopic observable: the volume of an extremal spacelike codimension-one slice connecting opposite sides of an eternal doublesided geometry. In two dimensions, this is simply the length of a geodesic. This observable has recently gained a lot of attention due to its ability to probe the horizon interior and also due to its connection to the notion of quantum computational complexity. ${ }^{2}$

Quantum computational complexity is a notion from quantum information which estimates the difficulty of constructing a quantum state from simple elementary operations [51, 52]. Complexity has some striking features which distinguish it from other measures of quantum correlations. Specifically, in chaotic systems, the complexity grows linearly following a quantum quench for a long time (exponential in the entropy of the system) and then saturates. It also reacts to perturbations in a characteristic way which encodes chaos and scrambling. All these behaviours have been reproduced using the maximal volume slices in AdS black holes. see e.g., [53-59].

These similarities led to conjecture that complexity of a quantum state is a plausible holographic dual to the extremal volume anchored at the boundary times where the state is defined

$$
\mathcal{C}_{V}=\max \frac{V}{G_{N} \ell}
$$

where $\ell$ is a certain length scale associated with the geometry, usually selected to be the AdS radius of curvature. ${ }^{3}$ In two-dimensional dilaton gravity it was suggested in [64] that equation (1.1) should include an additional factor $\Phi_{0}$ which is the constant part of the full dilaton field. Alternatively, propositions were made which relate the complexity to the action of the WdW patch $[65,66]$ and to its spacetime volume [67]. In cases with a horizon, all these quantities probe the behind horizon region. Here we explore the complexity=volume (CV) conjecture (1.1) in flow geometries.

We find that the geodesics in the flow geometries are strongly affected by the interior $\mathrm{dS}_{2}$ region and differ significantly from geodesics in $\mathrm{AdS}_{2}$ black holes. It is well known that spacelike geodesics in $\mathrm{dS}$ starting at a point will reach its antipodal point and that not all points on the worldline of an observer are connected by a spacelike geodesic with points on the "antipodal worldline". As a consequence, we will see that in most of our flow geometries not all boundary times will be connected by spacelike geodesics and only a finite

\footnotetext{
${ }^{2}$ Previous attempts to study quantum computational complexity in dS space appeared in [48-50].

${ }^{3}$ This conjecture was reformulated using Lorentzian flows in [60,61] similarly to the earlier reformulation of the Ryu-Takayanagi prescription [62] using 'bit threads' [63].
} 
short range of times of the order of the inverse temperature will have a complexity $=$ volume observable associated to it.

Another important difference is that, even when they exist, the length of geodesics does not behave as in the case of black holes. In the usual limit where the boundary lies very far from the horizon, the length of geodesics in the flow geometries with dS horizons behave as ${ }^{4}$

$$
\mathcal{C}_{V}(t) \sim S_{0} \log \cos \left(c_{\gamma} \pi T t\right)+\text { const }
$$

where $c_{\gamma}$ depends on the specific flow geometry we consider and $S_{0}$ is the entropy associated with the constant part of the dilaton. In most cases, the volume complexity decreases at early times reaching a minimum. This behaviour is valid up to times of the order of the inverse temperature $T t \lesssim 1 .^{5}$ At that point, there is a last geodesic that becomes (almost everywhere) null and reaches past/future infinity. At later times, finite-length geodesics cease to exist. A similar phenomenon was observed for pure dS geometries in [68]. This contrasts with the known result for the $\mathrm{AdS}_{2}$ black hole,

$$
\mathcal{C}_{V}(t) \sim S_{0} \log \cosh (\pi T t)+\text { const }
$$

where the volume complexity grows linearly at late times $t T \gg 1$. The above formula is a good approximation for the volume at all times as long as the boundary lies far enough from the horizon.

One might suspect that the different behaviours come from having glued together two geometries. In order to rule this out, we consider flow geometries that interpolate between an AdS black hole in the interior and an AdS with different curvature radius close to the boundary. In this case, we do recover exactly the same linear growth at late times as in the black hole case.

The rest of the paper is organised as follows: in section 2, we present the dilaton-gravity theories under consideration; in section 3 , we build the formalism to compute the length of the geodesics for an arbitrary geometry; section 4 discusses the known examples of some maximally symmetric spacetimes through this formalism; in section 5 , we compute the lengths of geodesics in different flow geometries that interpolate between an AdS boundary and different (A)dS interiors; we end up with a discussion of the different results in section 6 . Some details of the flow geometries have been relegated to two appendices.

\footnotetext{
${ }^{4}$ Slightly different behaviours can be obtained with different types of flow geometries with dS interiors as $c_{\gamma}$ becomes imaginary, but since the solutions are valid only for a short range of times, none of them produces linear growth at late times. See comments below and section 5.2.

${ }^{5}$ Equation (1.2) is a short time approximation valid for $T t \lesssim 1$ for large enough boundary-horizon separation and for most of the flow geometries we consider. Note that the expression becomes complex for times larger than $t_{\text {crit }}=1 /\left(2 c_{\gamma} T\right)$. Despite being a short time approximation, it captures many of the properties of the exact result. For example, the exact result for the volume also becomes imaginary at a time of the order of the inverse temperature.
} 


\section{$2 \quad 2 d$ dilaton-gravity theories}

We will study general dilaton-gravity theories in two dimensions. The Lorentzian action is given by,

$$
\begin{aligned}
S= & \frac{\Phi_{0}}{16 \pi G_{N}}\left(\int d^{2} x \sqrt{-g} R+2 \int d u \sqrt{-h} K\right) \\
& +\frac{1}{16 \pi G_{N}} \int d^{2} x \sqrt{-g}\left(\phi R+\ell^{-2} U(\phi)\right)+\frac{1}{8 \pi G_{N}} \int d u \sqrt{-h} \phi_{b}(K-1 / \ell) .
\end{aligned}
$$

The first term is topological and proportional to the Euler characteristic of the manifold. ${ }^{6}$ $K$ and $h$ are the extrinsic curvature and the induced metric on the boundary, respectively, $\ell$ is the curvature radius of the manifold, and $\phi_{b}$ is the value of the dilaton at the boundary. Finally, we require the full dilaton to be positive $\Phi=\Phi_{0}+\phi>0$ and we work in the limit where $\Phi_{0} \gg \phi$.

The equations of motion for the dilaton and the metric read

$$
\begin{aligned}
& R=-\frac{U^{\prime}(\phi)}{\ell^{2}}, \\
& 0=\nabla_{a} \nabla_{b} \phi-g_{a b} \nabla^{2} \phi+\frac{g_{a b}}{2 \ell^{2}} U(\phi) .
\end{aligned}
$$

Examples of such theories include the JT gravity theory where the dilaton potential is set to $U(\phi)=2 \phi$. For any sufficiently smooth dilaton potential, the equations of motion (2.2) admit solutions given by

$$
d s^{2}=-f(r) d t^{2}+\frac{d r^{2}}{f(r)}, \quad \phi=r / \ell
$$

where the blackening factor $f(r)$ is

$$
f(r)=\int_{r_{h} / \ell}^{r / \ell} U(\phi) d \phi
$$

and $r_{h}$ is the position of the event horizon where the blackening factor vanishes. It is straightforward to check that this solution satisfies the equations of motion for any potential. The thermodynamics of these dilaton gravity theories was studied in [34] (see also appendix A), where it was demonstrated that the temperature and entropy are given by

$$
T=\frac{U\left(\phi\left(r_{h}\right)\right)}{4 \pi \ell}, \quad S=\frac{\Phi_{0}+\phi\left(r_{h}\right)}{4 G_{N}} .
$$

The following derivations will not use the specific form of the blackening factor $f(r)$ from equation (2.4). Instead, we will use only the fact that our Lorentzian geometry takes the form (2.3) where $t \in \mathbb{R}$ and $f(r)$, for now, is a generic continuous function of the $r$-coordinate with AdS asymptotics, i.e., $f(r) \rightarrow r^{2}$ when $r \rightarrow \infty .^{7}$ We further assume

\footnotetext{
${ }^{6}$ Here we only focus on solutions which have a trivial topology, but see, e.g., [59] for the influence of different topologies on the complexity.

${ }^{7}$ In section 4.3 , we will relax this assumption when considering purely dS spacetime.
} 
that $f(r)$ has a single root within the physical range of the coordinate $r$ at some $r=r_{h}$ indicating the position of the horizon, that it is positive outside the horizon, and that the geometry can be maximally extended into a two-sided geometry with two boundaries.

In order to extend the geometry, it is useful to define a tortoise coordinate ${ }^{8}$

$$
r^{*}(r)=\int^{r} \frac{d \tilde{r}}{f(\tilde{r})} .
$$

Without loss of generality, we can choose the integration constant such that $r^{*}(r \rightarrow \infty)=0$, i.e., the tortoise coordinate vanishes at the AdS boundary. We next define lightcone coordinates,

$$
v_{R}=t_{R}+r^{*}, \quad u_{R}=t_{R}-r^{*} .
$$

The $R$ subscript indicates that these coordinates cover the right-side of the two-sided Penrose diagram. In these coordinates the metric (2.3) becomes

$$
d s^{2}=-f(r) d v_{R}^{2}+2 d v_{R} d r=-f(r) d u_{R}^{2}-2 d u_{R} d r .
$$

Similarly, we can define a set of left coordinates, $v_{L}=-t_{L}+r^{*}, u_{L}=-t_{L}-r^{*}$, that cover the left part of the Penrose diagram. Note that with this choice of coordinates, the time variable runs upwards along both boundaries. The different lightcone coordinates are depicted in figure 1 .

\subsection{Penrose diagram coordinates}

In order to draw a Penrose diagram it is convenient to define new coordinates $x^{+}, x^{-}$that are finite across the horizon. In the $x^{+}, x^{-}>0$ quadrant, these are defined by

$$
x^{+}=e^{v_{R} / \ell}, x^{-}=e^{-u_{R} / \ell} .
$$

Similar formulas apply in the other quadrants with some overall sign modifications. Note that $x^{+} x^{-}=e^{2 r^{*} / \ell}$, so constant $x^{+} x^{-}$correspond to constant- $r$ slices and similarly, constant $x^{+} / x^{-}=e^{2 t_{R} / \ell}$ corresponds to constant- $t_{R}$ slices. The $x^{ \pm}$coordinates still run from $-\infty$ to $\infty$, so to compactify them into the Penrose diagram we define $U_{R}, V_{R}$ coordinates such that

$$
x^{+}=\tan U_{R}, \quad x^{-}=\tan V_{R} .
$$

We will generally use coordinates where the asymptotic boundary of AdS is at $x^{+} x^{-}=1$, so that each boundary is a vertical line in the Penrose diagram.

\footnotetext{
${ }^{8}$ Some care has to be taken when evaluating the tortoise coordinate across the horizon. Under our assumptions, the blackening factor takes the form $f(r)=\left(r-r_{h}\right) F(r)$ where $F(r)$ has no other roots within the physical range of $r$. The inverse of $f(r)$ can be decomposed as

$$
\frac{1}{f(r)}=\frac{1}{F\left(r_{h}\right)\left(r-r_{h}\right)}+\frac{F\left(r_{h}\right)-F(r)}{F(r) F\left(r_{h}\right)\left(r-r_{h}\right)}
$$

which integrates to

$$
r^{*}(r)=\log \frac{\left|r-r_{h}\right|}{F\left(r_{h}\right)}+G(r)
$$

where $G(r)$ comes from integrating the second term on the right hand side of (2.6) and is completely regular at $r=r_{h}$.
} 


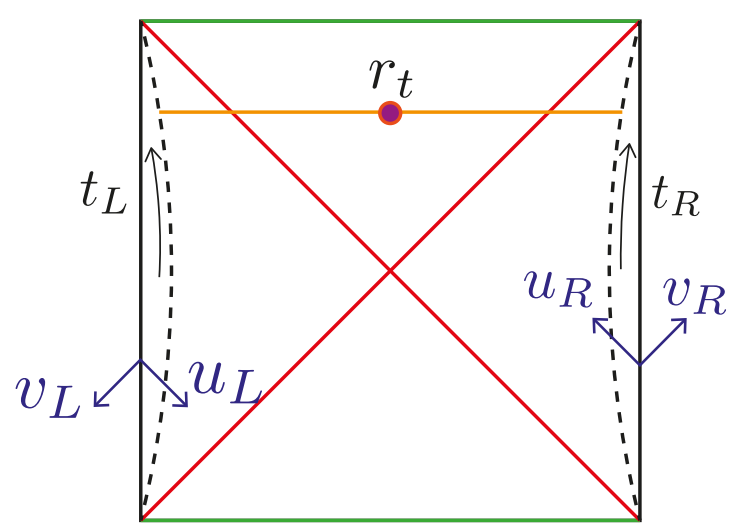

Figure 1. Penrose diagram for $\mathrm{AdS}_{2}$. The boundary $r=R_{b}$, corresponding to some fixed value of the dilaton $\phi=\phi_{b}$, is indicated by a dashed black line. The times $t_{L}=t_{R}$ run upwards along both boundaries. The axis of changing $u_{L / R}, v_{L / R}$ are indicated in the figure. We have also illustrated a geodesic with turning point $r_{t}$ (see below).

\section{Geodesics in 2d spacetimes}

The aim of this paper is to study the volume $e^{9}$ of spacelike geodesics that are anchored at fixed times on the two boundaries. It is possible to develop a formalism to find these geodesics and compute their volume for generic $f(r)$, see e.g., $[56,58]$. We explain how to do this in the current section. In the following sections, we will use this formalism to study the geodesics in specific examples including the flow geometries.

\subsection{Geodesics for general $f(r)$ geometries}

To find geodesics in geometries described in terms of a blackening factor $f(r)$, consider the volume of these geodesics

$$
V=\int d s \sqrt{-f \dot{v}_{R}^{2}+2 \dot{v}_{R} \dot{r}}=\int d s \sqrt{-f \dot{u}_{R}^{2}-2 \dot{u}_{R} \dot{r}},
$$

where $v_{R}, u_{R}$ and $r$ are parametrized in terms of a parameter $s$ and the dot indicates derivative with respect to this parameter. It is always possible to choose a parametrization where

$$
-f \dot{v}_{R}^{2}+2 \dot{v}_{R} \dot{r}=-f \dot{u}_{R}-2 \dot{u}_{R} \dot{r}=1,
$$

so that

$$
\dot{r}=\frac{1+f \dot{v}_{R}^{2}}{2 \dot{v}_{R}}=-\frac{1+f \dot{u}_{R}^{2}}{2 \dot{u}_{R}} .
$$

The volume does not depend explicitly on the $v_{R}\left(\right.$ or $u_{R}$ ) coordinate, so there is a conserved quantity

$$
P_{v}=\frac{\delta V}{\delta \dot{v}_{R}}=-f \dot{v}_{R}+\dot{r}=\frac{1-f \dot{v}_{R}^{2}}{2 \dot{v}_{R}}=-f \dot{u}_{R}-\dot{r}=\frac{1-f \dot{u}_{R}^{2}}{2 \dot{u}_{R}}=\frac{\delta V}{\delta \dot{u}_{R}}=P_{u} \equiv P .
$$

\footnotetext{
${ }^{9}$ The volume usually refers to the size of a codimension-one surface. In this paper we are interested in two dimensional geometries, so the volume is equivalent to the length of the geodesics. We will keep using the term volume throughout the rest of the text to be consistent with the complexity literature in higher dimensions.
} 
From here, we can solve for $\dot{v}_{R}$ and $\dot{u}_{R}$ as a function of $P$ and $r$ and obtain

$$
\dot{v}_{R \pm}=\frac{-P \pm \sqrt{f+P^{2}}}{f}=\dot{u}_{R \mp}
$$

which in turn implies using (3.4) that

$$
\dot{r}_{ \pm}= \pm \sqrt{f+P^{2}}
$$

Here, we see that we can interpret the subscripts \pm labelling the different solutions as an indication of whether the radial coordinate $r$ is increasing or decreasing with increasing $s$ along the geodesic.

At points where (3.6) changes sign the geodesic will turn around, i.e., if before this point the geodesic was moving away from the boundary into the interior of the geometry, after this point it will go back towards the boundary (in the second side of the double sided geometry). We denote the point where $\dot{r}_{ \pm}=0$ by $r_{t}$ and refer to it as the turning point, see figure 1 . It can be obtained by solving the equation

$$
f\left(r_{t}\right)+P^{2}=0 .
$$

Except for specific degenerate cases, all the geometries considered in this paper will admit a single turning point, i.e., equation (3.7) will have a single solution within the physical range of the coordinate $r$. The position of this turning point will depend on the form of $f(r)$ so we will discuss it later for each of the examples separately. In geometries with shockwaves $[54,58]$ multiple turning points can occur, but we will not be dealing with such cases here.

Next, let us write down expressions for the volume of the extremal slices. The isometries of our geometries imply that the volume is invariant under the following change of the boundary times $t_{R} \rightarrow t_{R}+\Delta t$ and $t_{L} \rightarrow t_{L}-\Delta t$ and hence only depends on the combination $t_{L}+t_{R}$. For simplicity, we will assume a symmetric configuration of the boundary times $t_{L}=t_{R}=t / 2$, but our result will be valid also for non-symmetric configurations. With the symmetric configuration, the total volume will be twice the volume on each side of the geometry. The latter is obtained by integrating (3.1) from the turning point towards the boundary along increasing $r$. We will assume that the boundary is fixed at some $\left.r\right|_{b d y}=R_{b}$ where the dilaton takes a constant value $\phi=\phi_{b}=R_{b} / \ell$, see eq. (2.3). Then,

$$
V[P]=\int d s=2 \int_{r_{t}}^{R_{b}} \frac{d r}{\dot{r}_{+}}=2 \int_{r_{t}}^{R_{b}} \frac{d r}{\sqrt{f(r)+P^{2}}} .
$$

This integral is well-behaved for any finite $R_{b}$. In particular, the integrand is smooth at the horizon.

Finally, we would like to relate the volume to the boundary times at which the geodesic is anchored. To find an expression for the boundary times in terms of the momentum $P$, we integrate eqs. (3.5)-(3.6) according to

$$
\begin{aligned}
& v_{R}\left(R_{b}\right)-v_{R}\left(r_{t}\right)=\int_{r_{t}}^{R_{b}} d r \frac{\dot{v}_{R+}}{\dot{r}_{+}}=\int_{r_{t}}^{R_{b}} \tau(P, r) d r, \\
& u_{L}\left(r_{t}\right)-u_{L}\left(R_{b}\right)=\int_{R_{b}}^{r_{t}} d r \frac{\dot{u}_{L-}}{\dot{r}_{-}}=\int_{r_{t}}^{R_{b}} \tau(P, r) d r,
\end{aligned}
$$


where

$$
\tau(P, r) \equiv \frac{\sqrt{f(r)+P^{2}}-P}{f(r) \sqrt{f(r)+P^{2}}} .
$$

In the above expressions, we have assumed that $P>0$. The geodesic moves from the left boundary to the right boundary, crossing behind the future horizon. Note that the integrand $\tau(P, r)$ does not diverge around $r=r_{h} \cdot{ }^{10} \quad$ Above, we have chosen the same boundary cutoff $R_{b}$ for the right and left boundaries.

Using the definition of the coordinates and summing up equations (3.9)-(3.10), we end up with

$$
\frac{t}{2}=r_{t}^{*}-r^{*}\left(R_{b}\right)+\int_{r_{t}}^{R_{b}} \tau(P, r) d r,
$$

where we have defined the total boundary time ${ }^{11}$

$$
t \equiv t_{L}+t_{R}
$$

The relations (3.8) and (3.13) are parametric equations for the volume and time in terms of the momentum $P$. Alternatively, inverting (3.13) gives $P(t)$, from which we can obtain $V(t)$.

Despite the somewhat complicated integrals which we will have to perform separately for each $f(r)$, it turns out that the rate of change of the volume has a very simple expression in terms of the momentum (cf. $[56,58])$ :

$$
\frac{d V}{d\left(t_{R}+t_{L}\right)}=P
$$

\subsection{Geodesics are always maximal}

By the upper semi-continuity of arc-length [69, chapter 9.4], spacelike geodesics, being extremal, will always have maximal volume, while timelike geodesics will always have maximal proper time. In our case, we can verify explicitly that the volume of the geodesics corresponds to a maximum. Consider the volume (3.1) in a parametrization set by the radial coordinate $s=r$. The the second variation of the volume functional $V[v(r)]$ with respect to the path $v(r)$ reads

$$
\delta^{(2)} V=-\int d r \frac{1}{\mathcal{V}^{3}} \delta v^{\prime}(r)^{2},
$$

where $\mathcal{V}=\sqrt{-f(r) v^{\prime}(r)^{2}+2 v^{\prime}(r)}$ is the (positive) volume element and $\delta v^{\prime}(r)=\frac{d}{d r} \delta v(r)$. (There is no term proportional to $\delta v(r)$ because the integrand only depends on $v^{\prime}(r)$.)

\footnotetext{
${ }^{10}$ We always parametrize our geodesics starting at the left boundary and ending on the right boundary. In this case $P>0$ corresponds to a geodesic crossing behind the future horizon which can be treated in terms of the $v_{R}$ and $u_{L}$ coordinates. The alternative case $P<0$ of geodesics passing behind the past horizon should be treated using the $u_{R}$ and $v_{L}$ coordinates. In the latter case, the time integral in eq. (3.13) will be replaced with

$$
\frac{t}{2}=-r_{t}^{*}+r^{*}\left(R_{b}\right)-\int_{r_{t}}^{R_{b}} \tau(-P, r) d r .
$$

Note that here too, the integrand does not diverge at the horizon. Most of the expressions we present in the following sections will be valid for both $P>0$ and $P<0$.

${ }^{11}$ Here $t_{L}$ and $t_{R}$ indicate the value of the time coordinate along the boundary curve $r=R_{b}$.
} 
Since the expression for $\delta^{(2)} V$ is manifestly negative, we conclude that the volume of geodesic corresponds to a maximum. We will see later situations where multiple geodesics correspond to some fixed boundary times and in these cases, all the geodesics will be of maximal length compared to nearby (non-extremal) trajectories.

\subsection{Working in dimensionless coordinates}

To simplify the notation in what follows, we will be using dimensionless coordinates. We redefine the radial coordinate $r_{d l}=r /\left|r_{h}\right|$, the dilaton potential $U(\phi)=U_{d l}\left(r_{d l}\right)\left|r_{h}\right| / \ell$ and the blackening factor $f(r)=f_{d l}\left(r_{d l}\right) r_{h}^{2} / \ell^{2}$ such that equation (2.4) becomes

$$
f_{d l}\left(r_{d l}\right)=\int_{\operatorname{sign}\left(r_{h}\right)}^{r_{d l}} U_{d l}\left(r_{d l}\right) d r_{d l} .
$$

Redefining a dimensionless volume $V_{d l}=V / \ell$, the volume integral (3.8) becomes

$$
V_{d l}\left[P_{d l}\right]=2 \int_{r_{d l, t}}^{R_{d l, b}} \frac{d r_{d l}}{\sqrt{f_{d l}(r)+P_{d l}^{2}}},
$$

where we have used the redefinitions $r_{d l, t}=r_{t} /\left|r_{h}\right|$ and $R_{d l, b}=R_{b} /\left|r_{h}\right|$ and redefined the momentum according to $P=P_{d l}\left|r_{h}\right| / \ell$. Finally, the time (3.13) reads

$$
\frac{t_{d l, R}+t_{d l, L}}{2}=r_{d l, t}^{*}-r_{d l}^{*}\left(R_{d l, b}\right)+\int_{r_{d l, t}}^{R_{d l, b}} \frac{\sqrt{f_{d l}\left(r_{d l}\right)+P_{d l}^{2}}-P_{d l}}{f_{d l}\left(r_{d l}\right) \sqrt{f\left(r_{d l}\right)+P_{d l}^{2}}} d r_{d l},
$$

where we have redefined the tortoise coordinate $(2.8) r^{*}(r)=r_{d l}^{*}\left(r_{d l}\right) \ell^{2} /\left|r_{h}\right|$, and the times $t=t_{d l} \ell^{2} /\left|r_{h}\right|$. Effectively, working in dimensionless conventions simply amounts to setting $\ell=\left|r_{h}\right|=1$ in all our previous formulas. From now on we will do so. We omit the $d l$ subscripts to keep the notation compact and keep in mind that in order to recover the dimensionful volume and time we should substitute

$$
V=\ell V_{d l}, \quad t=t_{d l} \ell^{2} /\left|r_{h}\right|
$$

\section{Geodesics in $\mathrm{A}(\mathrm{dS})$ spacetimes}

\subsection{Geodesics in global $\mathrm{AdS}_{2}$}

Solutions with constant negative curvature are obtained when $U(\phi)=2 \phi .^{12}$ The metric for global $\mathrm{AdS}_{2}$ is given by ${ }^{13}$

$$
f(r)_{\text {global }}=r^{2}+1 .
$$

The Penrose diagram is the infinite vertical strip. It has two boundaries and, in this coordinate system, $r$ runs from $r=-\infty$ at one boundary to $r=+\infty$ at the other. It is

\footnotetext{
${ }^{12}$ More generally, the Ricci scalar is give by $R=-f^{\prime \prime}(r)$.

${ }^{13}$ This geometry, which does not have a horizon, can be obtained by analytically continuing eq. (2.4) to imaginary horizon radius $r_{h}=i$.
} 


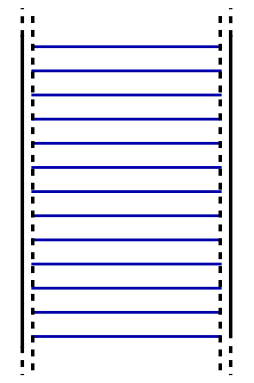

Figure 2. Penrose diagram for global $\mathrm{AdS}_{2}$. The geodesics in blue connect equal times on the two boundaries. The black dashed line is the cutoff surface $r=R_{b} \gg 1$.

known that geodesics at fixed, equal boundary times are just constant global time slices, as shown in figure 2. So it is straightforward to compute their volume,

$$
V=\int_{-R_{b}}^{R_{b}} \frac{d r}{\sqrt{f(r)_{\text {global }}}}=\left.\operatorname{arcsinh} r\right|_{-R_{b}} ^{R_{b}}=2 \log \left(2 R_{b}\right)+O\left(1 / R_{b}\right),
$$

where $R_{b}$ here serves as a UV regulator for the volume divergences near $|r|=\infty$. As expected, the volume is independent of the boundary times chosen. As a warm up exercise, we will use our general $f(r)$ procedure from section 3.1 to reproduce this result.

First, we note that in this geometry, geodesics do not have a turning point, i.e., $f(r)+P^{2}$ is always greater than zero, so instead of integrating from the turning point, we just integrate from one boundary to the other. The volume integral (3.8) gives

$$
V=\int_{-R_{b}}^{R_{b}} \frac{d r}{\sqrt{f(r)_{\text {global }}+P^{2}}} .
$$

To recover eq. (4.2), we need to show that $P=0$ for geodesics anchored at equal boundary times. To demonstrate this, we consider the time integral (3.9) and integrate from one boundary to the other

$$
v_{R}\left(R_{b}\right)-v_{R}\left(-R_{b}\right)=\int_{-R_{b}}^{R_{b}} \tau(P, r) d r
$$

Using the definition of $v_{R}(2.9)$, this can be re-expressed as

$$
t_{R}\left(R_{b}\right)-t_{R}\left(-R_{b}\right)=r^{*}\left(-R_{b}\right)-r^{*}\left(R_{b}\right)+2 \arctan \left(R_{b}\right)-2 \arctan \left(\frac{P R_{b}}{\sqrt{P^{2}+r^{2}+1}}\right) .
$$

The tortoise coordinate, vanishing at $r \rightarrow \infty$, is given by

$$
r^{*}(r)=\arctan r-\frac{\pi}{2} .
$$

With these ingredients, the requirement that the geodesics are anchored on both boundaries at the same time $t_{R}\left(R_{b}\right)=t_{R}\left(-R_{b}\right)$ yields

$$
0=-2 \arctan \left(\frac{P R_{b}}{\sqrt{P^{2}+R_{b}^{2}+1}}\right),
$$

which sets $P=0$. Then our volume integral (4.3) reduces to the one found in equation (4.2), as expected. 


\subsection{Geodesics in the $\mathrm{AdS}_{2}$ black hole}

The $\mathrm{AdS}_{2}$ black hole is actually very similar to the previous global $\mathrm{AdS}_{2}$. The difference is that the metric is expressed in Rindler coordinates and the boundary, located at some constant value of the Rindler radial coordinate, is bent towards the bulk in a way which makes parts of it inaccessible, i.e., hidden behind a horizon, see figure 1. The complexity of the $\mathrm{AdS}_{2}$ black hole was already studied in [64], where it was found that the geodesic length grows linearly with time at late times. This result is derived using the known fact that geodesics are lines of constant "global" time. Here, we reproduce that behaviour using the procedure described in section 3.1.

The $\mathrm{AdS}_{2}$ black hole is obtained once again using the dilaton potential $U(\phi)=2 \phi$. The corresponding blackening factor reads

$$
f(r)_{\mathrm{BH}}=r^{2}-1
$$

where we set the horizon and curvature radii $r_{h}=\ell=1$ as described in section 3.3. This corresponds to a temperature of $T=1 / 2 \pi$, see eq. (2.5). The boundary of AdS is at $r \rightarrow \infty$. In higher dimensional black holes there is a curvature singularity at $r \rightarrow 0$, but this is not the case in two dimensions.

We can follow the procedure outlined in the previous section. First, we need to find the turning point,

$$
f\left(r_{t}\right)+P^{2}=r_{t}^{2}-1+P^{2}=0 \rightarrow r_{t}=\sqrt{1-P^{2}},
$$

which gives a turning point $r_{t} \leq 1$ inside the horizon and implies that $-1 \leq P \leq 1$. Next we need to perform the volume and times integrals. The volume integral (3.8) can be performed analytically and yields

$$
V[P]=2 \operatorname{arccosh}\left(\frac{R_{b}}{\sqrt{1-P^{2}}}\right) .
$$

The time integral (3.13) can also be evaluated analytically. We first note that the tortoise coordinate $r^{*}(r)=\frac{1}{2} \log \left|\frac{r-1}{r+1}\right|$ at the turning point is given by

$$
r_{t}^{*}=-\operatorname{arccosh}\left(\frac{1}{|P|}\right) \text {. }
$$

Then, (3.13) becomes

$$
t=2 \operatorname{arctanh}\left(\frac{P R_{b}}{\sqrt{R_{b}^{2}-1+P^{2}}}\right) .
$$

Note that for large $R_{b}$, this expression gives $t_{L}+t_{R}=0$ for $P=0$ and $t_{L}+t_{R} \rightarrow \pm \infty$ for $P= \pm 1$, so it covers all boundary times. Luckily, it is also possible to invert this expression analytically and obtain

$$
P=\frac{\tanh \left(\frac{t}{2}\right) \sqrt{R_{b}^{2}-1}}{\sqrt{R_{b}^{2}-\tanh ^{2}\left(\frac{t}{2}\right)}} .
$$




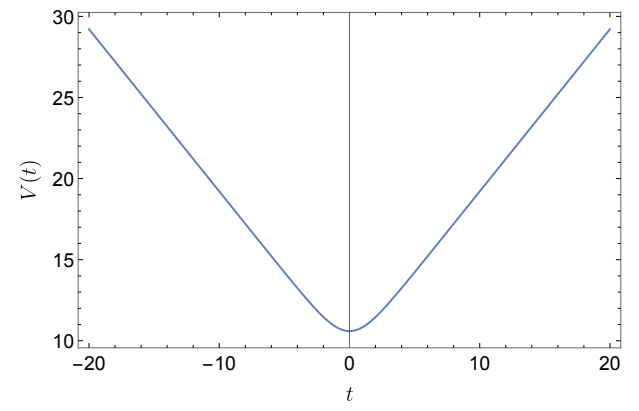

(a)

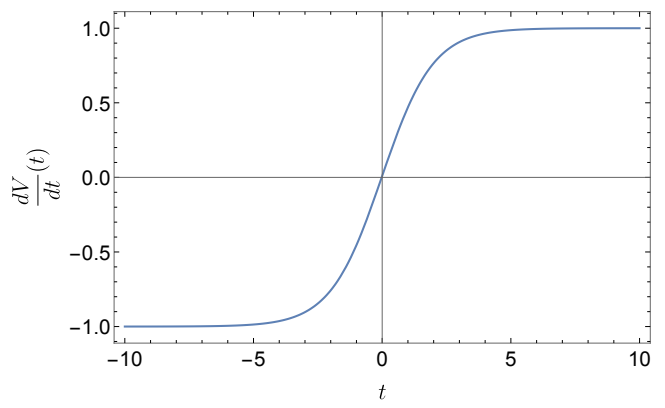

(b)

Figure 3. Volume and its time derivative as a function of the boundary time $t$ with $R_{b}=100$.

Plugging this into (4.10) we find that

$$
V(t)=2 \operatorname{arccosh}\left(\sqrt{\left(R_{b}^{2}-1\right) \cosh ^{2} \frac{t}{2}+1}\right) .
$$

We plot this function in figure 3 , where a linear growth at late times can be observed. In fact, if we expand this expression for large $R_{b}$, we obtain

$$
V(t)=2 \log \left(2 R_{b} \cosh \frac{t}{2}\right)+O\left(1 / R_{b}^{2}\right)
$$

which becomes at late times

$$
V(t) \approx 2 \log R_{b}+|t|+\cdots,
$$

which is the celebrated linear growth result. To eliminate the cutoff dependence, we may consider the time derivative of the volume

$$
\frac{d V}{d t}=\frac{\tanh \left(\frac{t}{2}\right) \sqrt{R_{b}^{2}-1}}{\sqrt{R_{b}^{2}-\tanh ^{2}\left(\frac{t}{2}\right)}}=P=\tanh \frac{t}{2}+O\left(1 / R_{b}^{2}\right) \underset{t \rightarrow \infty}{\longrightarrow} 1 .
$$

Recall that the equality to $P$ is a general property of the rate of change of the volume, see comments around equation (3.15). Re-establishing the dimensions using equation (3.20) and the thermodynamic quantities (2.5) we obtain in the late time limit

$$
\frac{d V}{d t}=r_{h} / \ell=2 \pi \ell T, \quad \frac{d \mathcal{C}_{V}}{d t}=8 \pi S_{0} T
$$

where the complexity was evaluated using eq. (1.1) with the extra factor of $\Phi_{0}$ suggested by [64], and $S_{0}$ is the leading contribution to the entropy.

We can instead consider only the part of the volume that lies behind the horizon. This requires integrating (3.8) from the turning point $r_{t}=\sqrt{1-P^{2}}$ to the horizon $r_{h}=1$, giving

$$
\begin{aligned}
V_{\text {inside }} & =2 \operatorname{arccosh}\left(\frac{1}{\sqrt{1-P^{2}}}\right) \\
& =2 \operatorname{arccosh}\left(\frac{\sqrt{\left(R_{b}^{2}-1\right) \cosh ^{2} \frac{t}{2}+1}}{R_{b}}\right)=|t|+O\left(1 / R_{b}^{2}\right) .
\end{aligned}
$$




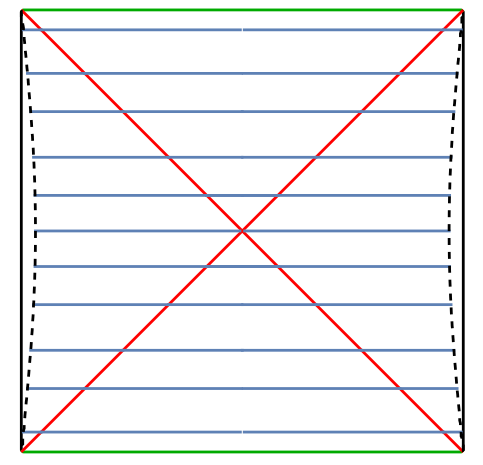

Figure 4. Penrose diagram for the $\mathrm{AdS}_{2}$ black hole and the geodesics in blue connecting equal times at the two boundaries. $R_{b}=10$ is the black dashed line.

Subtracting this from eq. (4.16), we see that the volume outside the horizon does not grow linearly and in fact approaches a constant at late times.

A collection of geodesics anchored at different boundary times in the $\mathrm{AdS}_{2}$ black hole Penrose diagram is shown in figure 4 . Note that the geodesics are indeed constant global time slices. ${ }^{14}$

\subsection{Geodesics in $\mathrm{dS}_{2}$}

In order to obtain solutions with positive constant curvature, we set $U(\phi)=-2 \phi$. The metric corresponds to pure $\mathrm{dS}_{2}$, with a blackening factor given by

$$
f(r) \mathrm{dS}=1-r^{2}
$$

We will concentrate on the part of the geometry with positive $r$. The radial coordinate outside the horizon ranges between $0 \leq r \leq 1$ and future/past infinity is reached as $r \rightarrow \infty$.

In this geometry there is no timelike boundary where it is natural to anchor the geodesics. Nonetheless, we can consider symmetric geodesics anchored at the observer's worldline $r=0$. Of course, these geodesics will not have a natural holographic interpretation at this point, but this calculation will provide some interesting intuition for the geodesics in the flow geometries which we consider in the next section. The technology is very similar to that developed in section 3.1. Geodesics in the $\mathrm{dS}_{2}$ spacetime have a turning point at

$$
r_{t}=\sqrt{1+P^{2}}
$$

and the tortoise coordinate is

$$
r^{*}(r)=\frac{1}{2} \log \left|\frac{r+1}{r-1}\right|
$$

\footnotetext{
${ }^{14}$ It is interesting to compare this shape of the geodesics to the extremal volumes obtained for higher dimensional black holes. In the latter case, the extremal slices wrap around constant $r_{\min }=r_{h} / 2^{1 / d}$ at late times, see section 3.1 of [56]. This is obtained by minimizing the turning point associated function $W(r)=\sqrt{-f(r)} r^{d-1}$, but since for us $d=1$ the minimization yields $r_{\min }=0$ and all the slices approach this straight line.
} 


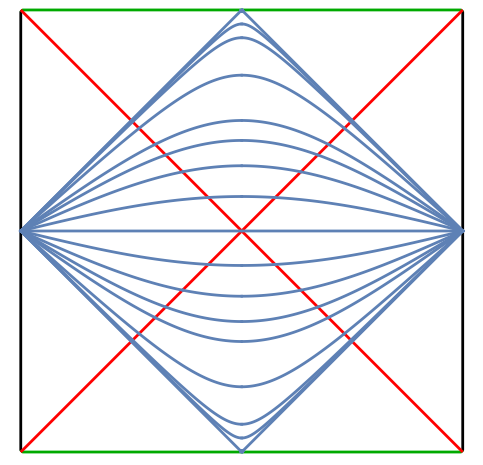

Figure 5. Penrose diagram for (half of) $\mathrm{dS}_{2}$ and the geodesics in blue connecting $t_{R}=t_{L}=0 . P$ runs from $-\infty$ to $\infty$ and in these limits, the geodesics become (almost everywhere) null.

with the integration constant chosen so that the tortoise coordinate vanishes on the observer's worldline $r^{*}(r=0)=0$. At the turning point this results in

$$
r_{t}^{*}=\operatorname{arcsinh}\left(\frac{1}{|P|}\right) \text {. }
$$

The volume integral (3.8) does not depend on $P$ and in fact, we obtain

$$
V[P]=\pi,
$$

for any spacelike geodesic in dS anchored at points with $r=0$. Evaluating the time integral, we obtain

$$
\frac{t_{R}+t_{L}}{2}=r_{t}^{*}+\int_{r_{t}}^{0} \tau(P, r) d r=0 .
$$

We see that geodesics anchored on the left and right $r=0$ worldlines must satisfy $t_{R}=-t_{L}$. This means that certain points on the worldlines are not connected by smooth spacelike geodesics of finite length. This can be seen from embedding $\mathrm{dS}_{2}$ in three dimensional Minkowski spacetime. In this picture, $\mathrm{dS}_{2}$ is the hyperboloid and geodesics are obtained by its intersections with planes that pass through the origin. In this embedding picture, it is clear that $t_{R}=-t_{L}$. The Penrose diagram with the symmetric $t_{L}=t_{R}=0$ geodesics are shown in figure 5 . We did not consider geodesics reaching future/past infinity whose lengths diverge. We will return to this point in section 6 . In the next section, we will use these results to compute the lengths of geodesics in flow geometries with a dS interior.

\section{Geodesics in flow geometries}

\subsection{Geodesics in the centaur geometry}

With our experience of $\mathrm{AdS}_{2}$ and $\mathrm{dS}_{2}$, we can now address the problem of finding geodesics in the centaur geometry

$$
f(r)_{\text {centaur }}= \begin{cases}\left(1-r^{2}\right), & -\infty<r<0 \\ \left(1+r^{2}\right), & 0<r<\infty\end{cases}
$$


This geometry is obtained as a solution of a dilaton-gravity theory with potential $U(\phi)=$ $2|\phi|{ }^{15}$ For large $r \rightarrow \infty$, the geometry looks like global $\mathrm{AdS}_{2}$. At $r=0$, it interpolates into a $\mathrm{dS}_{2}$ region with a horizon at $r=-1$. The choice of the horizon ${ }^{16}$ at $r_{h}=-1$ was made such that it gives the same temperature as for the black hole case $T=1 / 2 \pi$, see appendix A. We refer to this geometry as a centaur geometry.

We assume there is a turning point $r_{t}$ along our geodesics with

$$
r_{t}=-\sqrt{1+P^{2}} \text {. }
$$

Note that $r_{t}<0$, which implies that the turning point is inside the dS horizon. The next step is to define the tortoise coordinate. Again, we fix $r^{*}(r)$ so that it vanishes at the boundary and we require continuity along the interpolating curve $r=0$. Doing so, we obtain,

$$
r^{*}(r)_{\text {centaur }}= \begin{cases}\frac{1}{2} \log \left|\frac{r+1}{r-1}\right|-\frac{\pi}{2}, & -\infty<r<0, \\ \arctan (r)-\frac{\pi}{2}, & 0<r<\infty .\end{cases}
$$

Evaluating this at the turning point we further get

$$
r_{t}^{*}=-\frac{\pi}{2}-\operatorname{arcsinh}\left(\frac{1}{|P|}\right) .
$$

We can evaluate the volume and time integrals separating the integrals into intervals. Note that since the metric is continuous up to its first derivatives, there is no jump in $P$ along the geodesic. ${ }^{17}$ The volume integral (3.8) yields

$$
\begin{aligned}
V[P] & =2\left(\int_{\mathrm{dS}}+\int_{\mathrm{AdS}}\right) \frac{d r}{\sqrt{f(r)_{\text {centaur }}+P^{2}}} \\
& =2\left(\int_{r_{t}}^{0} \frac{d r}{\sqrt{1-r^{2}+P^{2}}}+\int_{0}^{R_{b}} \frac{d r}{\sqrt{1+r^{2}+P^{2}}}\right) \\
& =\pi+2 \operatorname{arcsinh}\left(\frac{R_{b}}{\sqrt{P^{2}+1}}\right) .
\end{aligned}
$$

Note that the $\pi$ contribution comes precisely from the dS part and it is the same that we got in section 4.3. The second term is the contribution from the AdS patch. Similarly, we can perform the time integral,

$$
\begin{aligned}
\frac{t}{2}= & r_{t}^{*}-r^{*}\left(R_{b}\right)+\int_{r_{t}}^{0} \tau_{\mathrm{dS}}(P, r) d r+\int_{0}^{R_{b}} \tau_{\mathrm{AdS}}(P, r) d r \\
= & r_{t}^{*}-r^{*}\left(R_{b}\right)+\left.\frac{1}{2} \log \left(\left|\frac{(r+1)\left(\sqrt{P^{2}-r^{2}+1}-P r\right)}{(r-1)\left(P r+\sqrt{P^{2}-r^{2}+1}\right)}\right|\right)\right|_{r_{t}} ^{0} \\
& +\left.\left(\arctan (r)-\arctan \left(\frac{P r}{\sqrt{P^{2}+r^{2}+1}}\right)\right)\right|_{0} ^{R_{b}} \\
= & -\arctan \left(\frac{P R_{b}}{\sqrt{R_{b}^{2}+P^{2}+1}}\right)=-\arctan P+O\left(1 / R_{b}^{2}\right) .
\end{aligned}
$$

\footnotetext{
${ }^{15}$ One might worry that this potential has a discontinuity in its derivative. It has been shown in [34] that this is not a problem, since it can be thought of as a smooth limit of continuous dilaton potentials.

${ }^{16}$ Recall that in two dimensions there are two cosmological horizons at $r_{h}= \pm 1$.

${ }^{17}$ This can be proven using the equations of motion for the volume (3.1) integrated in a small shell around the transition between the dS and the AdS regions.
} 


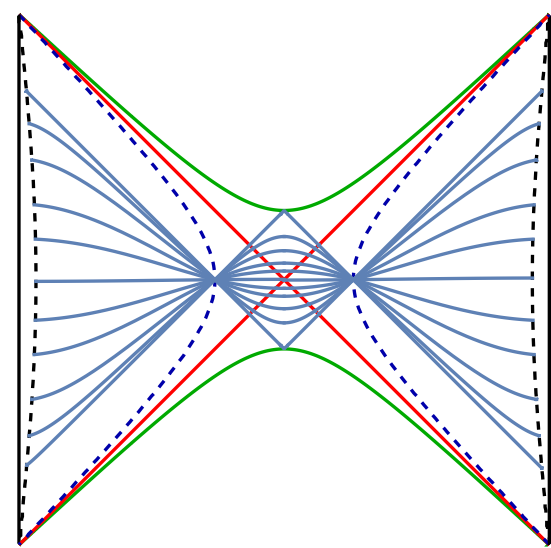

(a) $P= \pm(100,2,1,0.5,0.25,0.01)$.

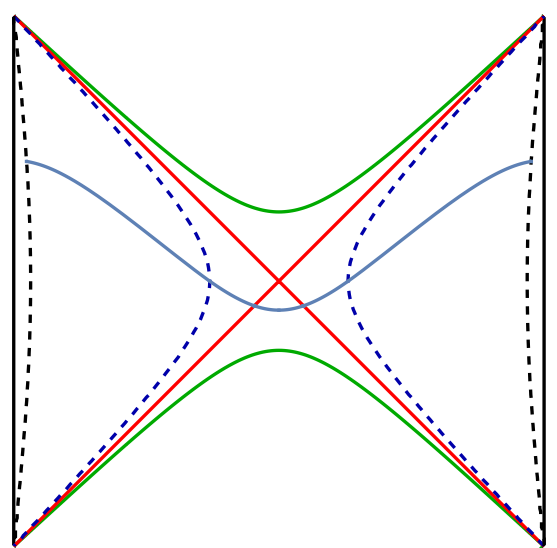

(b) $P=-1$.

Figure 6. Penrose diagrams for the centaur geometry and geodesics anchored at different boundary times in blue, spanning the full range of times for which smooth spacelike geodesics of finite length exist. The dashed black line is the cutoff surface with $R_{b}=10$. The dark blue dashed line is the interpolating line between the two geometries at $r=0$. The red lines correspond to the horizons and the green ones correspond to $r \rightarrow-\infty$.

It is interesting to note that for positive $P$, the times are negative (and vice versa) and also that while $-\infty<P<\infty$, the times are constrained to the range $-\pi<t<\pi$, so it is not possible to obtain geodesics connecting boundary points at equal arbitrarily large times. This can be appreciated in the Penrose diagram in figure $6 \mathrm{a}$ where we plot some of the geodesics.

The reason for this is that in the dS part, the only allowed geodesics start at $t=0$ and not all boundary points in the AdS part are spacelike connected to this point. It would be interesting to understand this intriguing feature of the geometry from the boundary quantum perspective. We return to this point in section 6 .

Another important difference between the centaur geometry and the AdS black hole is that for the centaur, geodesics anchored at positive boundary times pass through the past horizon, and not the future one, as can be seen from figure $6 \mathrm{~b}$.

Finally, we can express the volume in terms of the boundary times. For this, we first need to invert equation (5.6) which yields

$$
P^{2}=\frac{\left(R_{b}^{2}+1\right) \tan ^{2}\left(\frac{t}{2}\right)}{R_{b}^{2}-\tan ^{2}\left(\frac{t}{2}\right)}
$$

as long as $R_{b}>\tan \frac{|t|}{2}$. Inserting this into equation (5.5) we get

$$
V(t)=\pi+2 \operatorname{arcsinh}\left(\sqrt{\left(R_{b}^{2}+1\right) \cos ^{2}\left(\frac{t}{2}\right)-1}\right),
$$

and at large $R_{b}$ this becomes

$$
V(t)=\pi+2 \log \left(2 R_{b} \cos \frac{t}{2}\right)+O\left(1 / R_{b}^{2}\right),
$$


which is valid as long as $-\pi<t<\pi$ and $R_{b}-\tan \frac{|t|}{2} \gg 0 .^{18}$ It is straightforward to compute the time derivative,

$$
\frac{d V(t)}{d t}=P=-\frac{\sqrt{R_{b}^{2}+1}}{\sqrt{R_{b}^{2}-\tan ^{2}\left(\frac{t}{2}\right)}} \tan \left(\frac{t}{2}\right)=-\tan \frac{t}{2}+O\left(1 / R_{b}^{2}\right) .
$$

Re-establishing the dimensions using eq. (3.20) and the thermodynamic quantities (2.5) we obtain

$$
\frac{d V}{d t}=-2 \pi \ell T \tan (\pi t T), \quad \frac{d \mathcal{C}_{V}}{d t}=-8 \pi S_{0} T \tan (\pi t T),
$$

where the complexity was evaluated using eq. (1.1) with the extra factor of $\Phi_{0}$ suggested by [64], and $S_{0}$ is the leading contribution to the entropy.

Plots of these functions can be found in figure 7 . The behaviour exhibited by the centaur geometry is radically different from the one observed in the black hole case, even though both geometries have an event horizon. Comparing equations (4.15) and (5.9), we note that they are related by changing $t \rightarrow i t$. Nevertheless, their behaviour is completely different. The centaur geometry does not exhibit linear growth of the volume as a function of time and in fact, there is no growth at all but a decrease in volume as time advances. While the time derivative of the volume in the black hole case goes to a constant, here it diverges when approaching the edges of the range of allowed times. Moreover, the length of the geodesic in the dS part of the geometry remains constant at a value of $\pi$. Finally, this behaviour is not valid for arbitrary long times. After a certain time, there are no connected geodesics between the two boundaries of spacetime.

It is instructive to compute the part of the volume that lies behind the horizon. This requires integrating from the turning point $r_{t}=-\sqrt{1+P^{2}}$ to the horizon $r_{h}=-1$. We find:

$$
V_{\text {inside }}[P]=2 \int_{-\sqrt{1+P^{2}}}^{-1} \frac{d r}{\sqrt{1-r^{2}+P^{2}}}=\pi-2 \arcsin \left(\frac{1}{\sqrt{1+P^{2}}}\right) .
$$

Since the geodesic is still anchored to the boundary, we can still use (5.6) to associate a time to it. This yields a very short regime of linear growth

$$
V_{\text {inside }}=\pi-2 \arcsin \left(\frac{\sqrt{\left(1+R_{b}^{2}\right) \cos ^{2} \frac{t}{2}-1}}{R_{b}}\right)=|t|+O\left(1 / R_{b}^{2}\right),
$$

valid for times $|t|<\pi$, as follows from (5.6) with $-\infty<P<\infty$. In contrast, in this range of times, the geodesic length inside the black hole grows quadratically with time, as can be seen from expanding equation (4.15).

\subsection{Geodesics in $\gamma$-centaurs}

The centaur geometry from the previous section is one out of a larger family of flow geometries that interpolate between an AdS boundary and a dS interior. This family

\footnotetext{
${ }^{18}$ Note that the volume of the last geodesic at $t= \pm 2 \arctan R_{b}$ can be extracted from (5.8) and is given by $\pi$ as expected.
} 


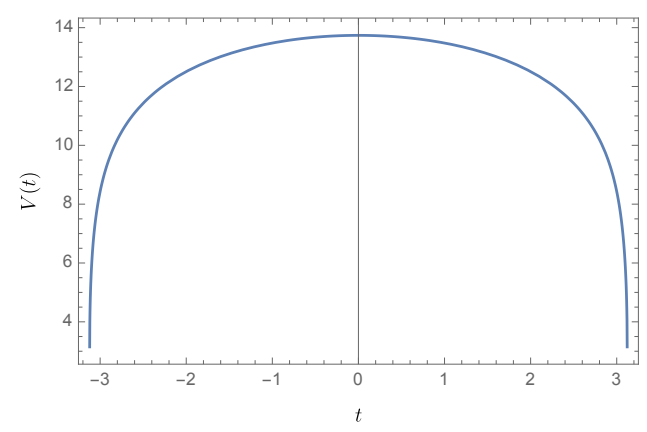

(a)

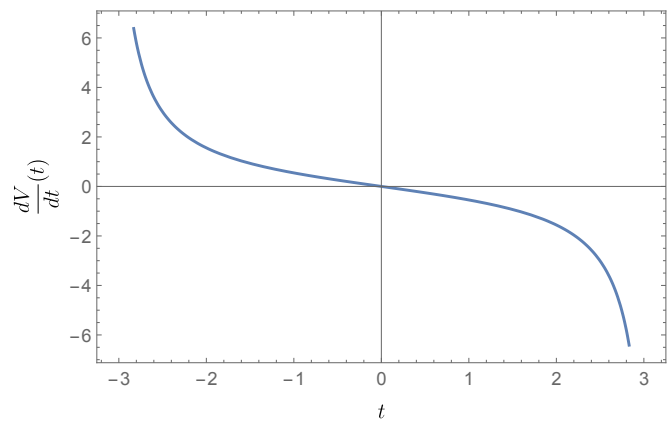

(b)

Figure 7. Volume and its time derivative as a function of the boundary time $t$ for the centaur geometry with $R_{b}=100$.

is characterized by a continuous parameter representing the radial location where the dS space is glued to AdS. While the centaur geometry is the most symmetric example, all these geometries are equally important to understand the behaviour of the cosmological horizon. In this section we would like to employ those geometries to check how our results depend on this gluing point.

The continuous flow geometries that sharply interpolate between $\mathrm{AdS}_{2}$ and $\mathrm{dS}_{2}$ can be parametrized by a single real parameter $\gamma \in[-1,1]$. We will refer to them as $\gamma$-centaur geometries. They can be thought of as solutions of a different dilaton-gravity theory with a generalized dilaton potential $U(\phi)=2\left(\left|\phi-\phi_{0}\right|-\phi_{0}\right)$, as shown in appendix D of [35]. The parameters $\phi_{0}$ and $\gamma$ are related according to

$$
\gamma \equiv 1-2 \phi_{0}^{2}
$$

The thermodynamic properties of these theories are explored in appendix A.

Using (2.4), the metric in the Schwarzschild gauge which we will be using, takes the following form ${ }^{19}$

$$
f(r)_{\gamma}= \begin{cases}1-r^{2}, & -\infty<r<\phi_{0} \\ 1+r^{2}+2 \phi_{0}\left(\phi_{0}-2 r\right), & \phi_{0}<r<\infty\end{cases}
$$

where we again set $r_{h}=-1$, to keep the same temperature as before. For $r<\phi_{0}$, the geometry has positive curvature while for $r>\phi_{0}$, the curvature is negative. We therefore see that $\phi_{0}$ corresponds to the (radial) gluing location between the two geometries. The parameters $\phi_{0}$ and $\gamma$ can lie in the following ranges

$$
\begin{cases}\frac{1}{\sqrt{2}}>\phi_{0}>0 & \rightarrow 0<\gamma<1 \\ 0>\phi_{0}>-\frac{1}{\sqrt{2}} & \rightarrow 1>\gamma>0 \\ -\frac{1}{\sqrt{2}}>\phi_{0}>-1 & \rightarrow 0>\gamma>-1\end{cases}
$$

\footnotetext{
${ }^{19}$ While $\phi_{0}$ is a natural variable in the Schwarzschild gauge, in the conformal gauge it is more convenient to use $\gamma$. The relation between the two coordinate systems is given in appendix B.
} 
where $\phi_{0}=0$ (or $\gamma=1$ ) corresponds to the centaur geometry where AdS and dS are glued exactly at $r=0$, and where $\phi_{0}=-1$ (or $\gamma=-1$ ) corresponds to a case where the interpolating region sticks to the horizon, so that outside the horizon there is only negatively-curved spacetime. Positive $\phi_{0}$ solutions correspond to having a larger part of the dS spacetime while negative $\phi_{0}$ corresponds to having a smaller dS portion.

Having the form of $f(r)$, we can now proceed to evaluate the integral expressions for the volume and boundary time. While they can be found analytically, some of the expressions are quite cumbersome and we therefore relegate the derivation and exact results for the volume and time as a function of the momentum $(t[P], V[P])$ to appendix $\mathrm{C}$, see equations (C.5)-(C.6). Here, we will discuss only their general properties and present interesting limits. The main results from our analysis (as we will see below) are the following.

(A) There do not exist geodesics beyond a certain boundary time of the order of the inverse temperature. In the range $-1<\phi_{0}<-\frac{1}{\sqrt{2}}$, for several boundary times there exist two or three different geodesics anchored at the same time.

(B) For the range $\left|\phi_{0}\right|<1 / \sqrt{2}$, the volume decreases as a function of time, until a time of the order of the inverse temperature, where the geodesics stop existing. In the range $-1<\phi_{0}<-\frac{1}{\sqrt{2}}$ there is a short period of quadratic increase in the maximal volume but even in this case, the maximal geodesics stop existing at times of the order of the inverse temperature.

(A) Behaviour of the boundary times. For $\left|\phi_{0}\right|<\frac{1}{\sqrt{2}}$ the behaviour is similar to that of the centaur geometry in the previous section, where each boundary time has at most one specific geodesic associated with it. However, those geodesics only exist for times in the range ${ }^{20}$

$$
|t| \leq \frac{1}{\sqrt{1-2 \phi_{0}^{2}}}\left(\pi+2 \arctan \left(\frac{\phi_{0}}{\sqrt{1-2 \phi_{0}^{2}}}\right)\right)-\log \left(\frac{1-\phi_{0}}{1+\phi_{0}}\right)+O\left(1 / R_{b}\right),
$$

where, of course, for $\phi_{0}=0$, this gives the range $|t|<\pi$ as in the previous section. Moreover, as $\left|\phi_{0}\right| \rightarrow \frac{1}{\sqrt{2}}$, the time gap goes to infinity, allowing geodesics at all times. In this case the $\gamma \rightarrow 0^{+}$centaur geometry develops an infinitely long AdS throat, see [35]. This will be evident later when plotting the volumes.

In contrast, for the range $-1<\phi_{0}<-\frac{1}{\sqrt{2}}$, at very early times, there are three different geodesics anchored at the very same boundary time. As we will see later, they also have different lengths. Depending on the value of $\phi_{0}$, at later times, there are two geodesics for a given boundary time or only one, see figure 8 . At larger times, of the order of the inverse temperature, the geodesics stop existing, see figure 9.

(B) Behaviour of the volume. As mentioned above, in the appendix we provide exact analytic results for the volume and boundary time as a function of the momentum $(V[P], t[P])$ (see equations (C.5)-(C.6)). Of course, these provide a parametrization of the volume as a function of time $V(t)$, but an analytic expression cannot be found for arbitrary $\phi_{0}$. Nevertheless, we can plot $V(t)$ numerically, for various values of $\phi_{0}$, see figure 10 .

\footnotetext{
${ }^{20}$ Introducing back the dimensional quantities, the left hand side is replaced with $2 \pi T|t|$.
} 


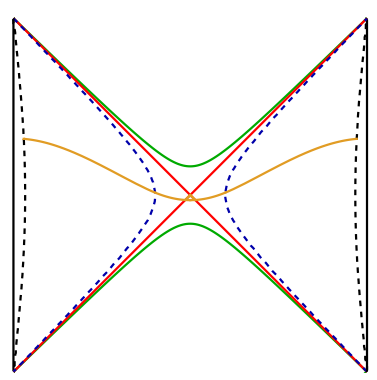

(a) $\phi_{0}=0.2$

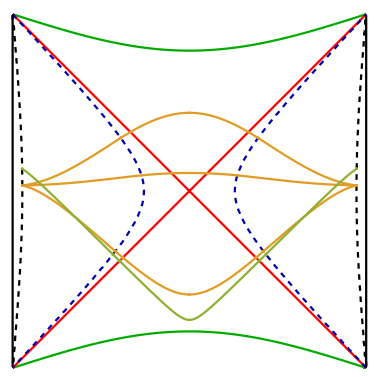

(b) $\phi_{0}=-0.85$

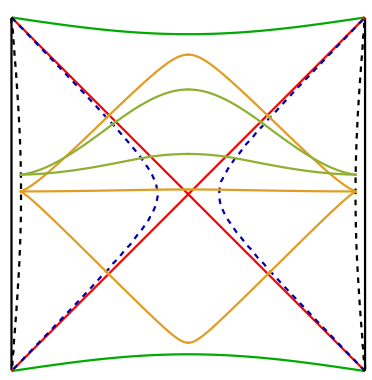

(c) $\phi_{0}=-0.95$

Figure 8. Penrose diagrams for the different $\gamma$-centaur geometries and geodesics anchored at different boundary times. The dashed black line is the cutoff surface with $R_{b}=10$. The dark blue dashed line is the interpolating line at $r=\phi_{0}$, the red lines are the horizons and the green ones are $r \rightarrow-\infty$. Yellow lines correspond to geodesics at early times and green lines to geodesics at slightly later times.

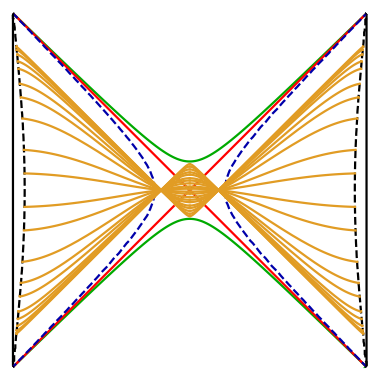

(a) $\phi_{0}=0.2$

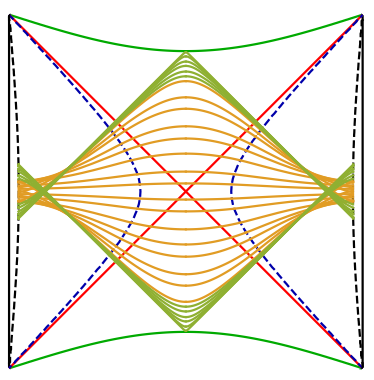

(b) $\phi_{0}=-0.85$

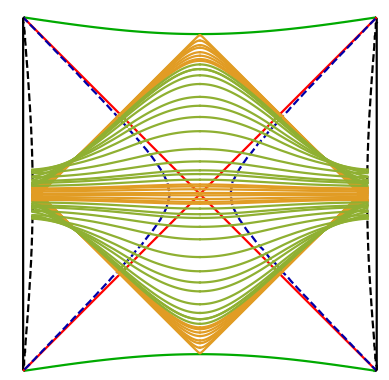

(c) $\phi_{0}=-0.95$

Figure 9. Same Penrose diagrams as in figure 8, but here we plot geodesics for several different boundary times. Note that the geodesics stop existing for large times.

The volume shows different behaviours depending on the value of $\phi_{0}$. For the range $\left|\phi_{0}\right|<1 / \sqrt{2}$, the form is similar to the one we found for the centaur in the previous section: the volume starts at a maximum at $t=0$ and decreases for some time until there are no more geodesics. This can be seen in the green, red and yellow curves in figure 10a. For the range $-1<\phi_{0}<-1 / \sqrt{2}$, the behaviour changes due to the existence of two or three geodesics anchored at the same time. This range corresponds to the blue curves in figure 10a, or the enlarged version in figure 10b. Indeed, we see that the blue curves represent a multivalued function with, at times, two or three different volumes corresponding to a given boundary time. The geodesic with largest volume (the uppermost blue curve) quadratically increases at very early times, similarly to what happens for black holes, but this behaviour never turns to a linear increase. This is consistent with the fact that the interior geometry does not have a black hole like behaviour and in fact, the geodesics inside the dS region never grow to size larger than $2 \pi$. So we do not expect to find linear growth at late times, even though outside the horizon, most of the geometry looks like the AdS black hole. We return to this point in section 6 . 


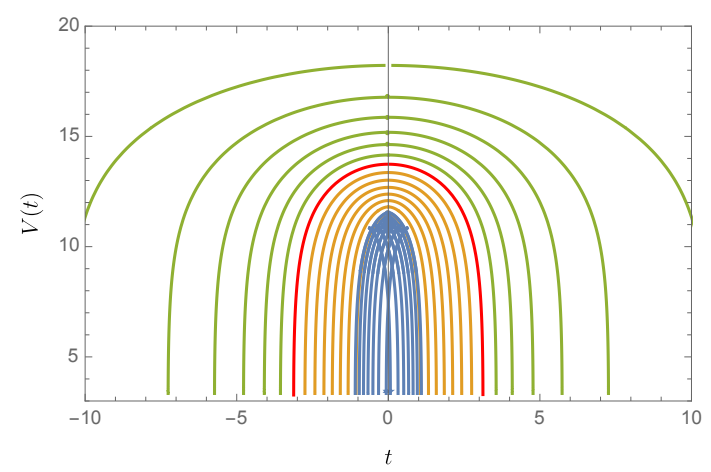

(a)

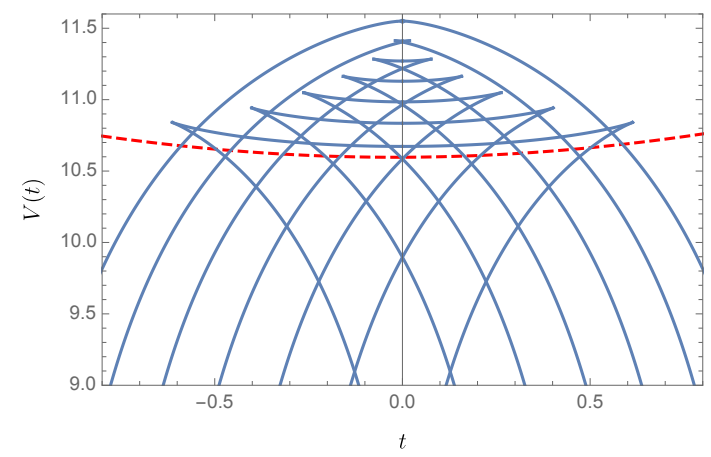

(b)

Figure 10. Volume as a function of the boundary time $t$ for the different $\gamma$-centaur geometries with $R_{b}=100$. In (a), the outermost green curve corresponds to $\phi_{0}=0.7$ and adjacent green, red and yellow curves correspond to decreasing $\phi_{0}$ in jumps of -0.1 up to $\phi_{0}=-0.7$. The green curves correspond to $\phi_{0}>0$, and the yellow ones to $\phi_{0}<0$. The thicker red curve $\phi_{0}=0$, corresponds to the centaur geometry from the previous section. For smaller $\phi_{0}$ we plotted curves in blue from $\phi_{0}=-0.74$ to $\phi_{0}=-0.99$ in steps of -0.05 . In (b), we zoom in this last region, showing the different behaviour of curves with $\phi_{0}<-1 / \sqrt{2} \sim-0.7$. In dashed red, we show the analytic result for the AdS black hole and we see how as $\phi_{0}$ approaches -1 , the curves tend to the one of the black hole, but only for short times. They never admit linear growth.

When more than one geodesic exists at a given time, in order to compute the complexity, we need to use the one with maximal volume. If we follow some of the blue curves in figure 10b along increasing time starting at $t=0$ and always pick the branch of maximal volume, we see two types of behaviours. The maximal volume for values of $\phi_{0}$ slightly below $-1 / \sqrt{2}$ will start increasing quadratically. It will then jump discontinuously to a lower value and start deceasing. This decrease will stop at some time of the order of the inverse temperature when the curves stop existing. For values of $\phi_{0}$ closer to -1 , the maximal volume will initially increase quadratically (again, just for a short time) and then the curves will stop existing. The transition between these two behaviours happens at $\phi_{0} \sim-0.928$.

While we do not have a closed form analytic expression for the volume as a function of time, such an expression can be found in an expansion around $t=0 .{ }^{21}$ The result obtained (up to quadratic order in $t$ ) can be recast in the following form, for the entire range of $\phi_{0},{ }^{22}$

$$
\begin{aligned}
V_{ \pm}(t)= & \pi \pm 2 \arctan \left(\frac{\sqrt{1-\gamma}}{\sqrt{1+\gamma}}\right)-2 \log \left(\frac{\sqrt{1+\gamma} \mp \sqrt{1-\gamma}}{\sqrt{2}}\right)+ \\
& 2 \log \left(2 R_{b} \cos \frac{\sqrt{1 \mp \sqrt{1-\gamma^{2}}}}{\sqrt{\gamma}} \frac{t}{2}\right)+O\left(1 / R_{b}\right)
\end{aligned}
$$

where $V_{ \pm}$corresponds to $\phi_{0}>0$ and $\phi_{0}<0$, respectively. This expression is in fact a good

\footnotetext{
${ }^{21}$ This is done by expanding the parametrization $(V[P], t[P])$ around $P=0$ and using the expansion to obtain an analytic expression for $V(t)$.

${ }^{22}$ This yields equation (1.2) with the identification $c_{\gamma}=\frac{\sqrt{1 \mp \sqrt{1-\gamma^{2}}}}{\sqrt{\gamma}}$.
} 
approximation for the volume up to times $T t \lesssim c$, where $c$ is some order one constant. In the case $-1<\phi_{0}<-1 / \sqrt{2}$ this expression only refers to the uppermost branch of the volume, see figure 10b. This expression encodes the fact that at early times the volume is quadratic in $t$. Note that for $\phi_{0}=0(\gamma=1)$ we recover the centaur result in equation (5.9) and for $\phi_{0}=-1(\gamma=-1)$, the expression becomes that of the AdS black hole - see equation (4.15). We stress again that though equal, this expression does not hold for arbitrary long times, as in the black hole case. It is interesting to see that for $\gamma<0$, the cos turns into a cosh, generating the change in behaviour found when going from the yellow curves to the blue ones in figure 10a. Finally, note that as $\gamma \rightarrow 0$ with positive $\phi_{0} \rightarrow 1 / \sqrt{2}$, the value of the volume at $t=0$ diverges logarithmically in $\gamma$. This trend is reflected in the increasing time span of the uppermost green curves in figure 10. This divergence is independent of the time. Recall that in this case the AdS part of the geometry develops an infinitely long throat, see appendix D of [35].

\subsection{Geodesics in AdS-to-AdS geometries}

The last case we will analyse is gluing two AdS-like spaces with different radii, in order to highlight the differences with the previous AdS-to-dS case.

As with the AdS-to-dS case, it is possible to construct dilaton-gravity theories in two dimensions, whose solutions interpolate between two AdS spacetimes with different curvature radii. The dilaton potential is given by

$$
U(\phi)_{\text {AdS-to-AdS }}= \begin{cases}2 \phi, & \phi<\phi_{0}, \\ (2+\alpha) \phi-\alpha \phi_{0}, & \phi>\phi_{0},\end{cases}
$$

in terms of two parameters $\phi_{0}$ and $\alpha$. The parameter $\phi_{0}$ fixes the location of the interpolation. We will assume it is greater than the horizon radius ( $r_{h}=1 \mathrm{in}$ our conventions), so the transition is outside the horizon. The second parameter $\alpha$ characterizes the radius of the second AdS. It ranges between $-2<\alpha<\infty$, where $\alpha=-2$ corresponds to an interpolation to flat spacetime and $\alpha=0$ corresponds to no interpolation at all, see figure 11 .

The interior AdS has unit radius and we will set the horizon at $r_{h}=1$. Then the metric becomes,

$$
f(r)_{\text {AdS-to-AdS }}= \begin{cases}\left(r^{2}-1\right), & 0<r<\phi_{0}, \\ r^{2}-1+\frac{1}{2} \alpha\left(r-\phi_{0}\right)^{2}, & \phi_{0}<r<\infty .\end{cases}
$$

The procedure is identical to the other cases, so we will just state here the main results. The turning point is

$$
r_{t}=\sqrt{1-P^{2}}
$$

corresponding to a range $-1<P<1$ of the conserved momentum. The tortoise coordinate is

$$
r^{*}(r)_{\text {AdS-to-AdS }}= \begin{cases}-\frac{1}{2} \log \left|\frac{r+1}{r-1}\right|+c_{1}, & 0<r<\phi_{0}, \\ \frac{\arctan \left(\frac{(\alpha+2) r-\alpha \phi_{0}}{\sqrt{2 \alpha\left(\phi_{0}^{2}-1\right)-4}}\right)-\pi / 2}{\sqrt{\frac{1}{2} \alpha\left(\phi_{0}^{2}-1\right)-1}}, & \phi_{0}<r<\infty,\end{cases}
$$



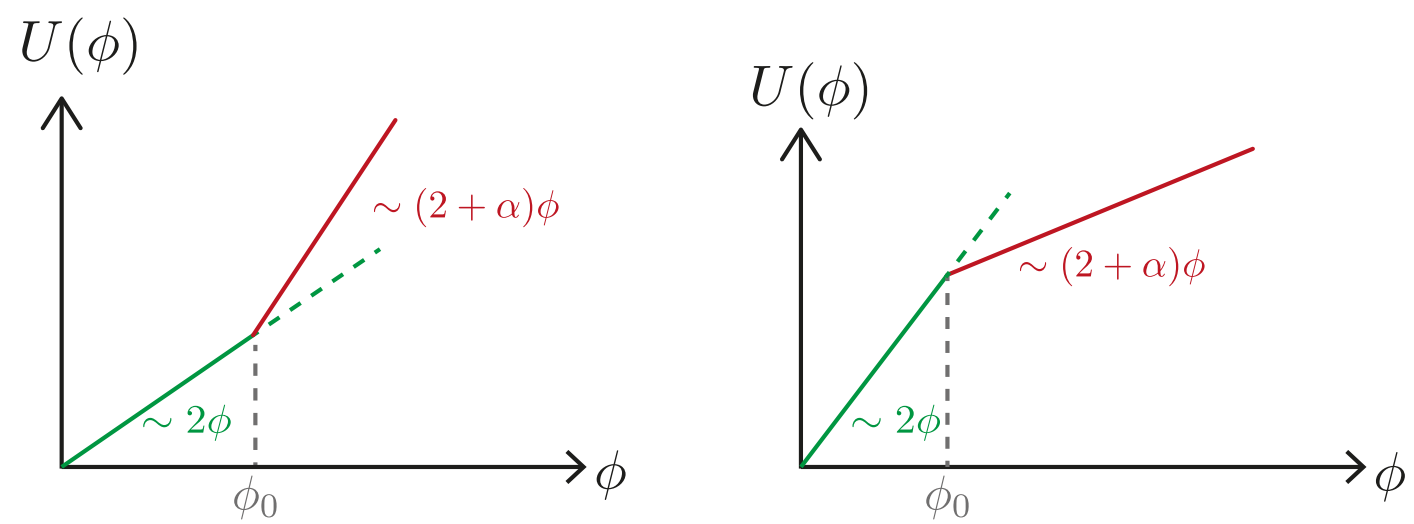

Figure 11. The AdS-to-AdS potential for $\alpha>0$ (left) and $\alpha<0$ (right). The crossing between the two regimes happens at $\phi=\phi_{0}$.

where the constant $c_{1}$ is given by

$$
c_{1}=\frac{2 \arctan \left(\frac{\phi_{0}}{\sqrt{\frac{1}{2} \alpha\left(\phi_{0}^{2}-1\right)-1}}\right)-\pi}{\sqrt{2\left(\alpha \phi_{0}^{2}-\alpha-2\right)}}-\frac{1}{2} \log \left(\frac{\phi_{0}-1}{\phi_{0}+1}\right) .
$$

At the turning point, this becomes

$$
r_{t}^{*}=\frac{\arctan \left(\frac{\phi_{0}}{\sqrt{\frac{1}{2} \alpha\left(\phi_{0}^{2}-1\right)-1}}\right)-\pi / 2}{\sqrt{\frac{1}{2} \alpha\left(\phi_{0}^{2}-1\right)-1}}+\operatorname{arctanh}\left(\frac{\sqrt{1-P^{2}} \phi_{0}-1}{\sqrt{1-P^{2}}-\phi_{0}}\right) .
$$

The volume integral gives

$$
V[P]=\log \left(\frac{\phi_{0}+\sqrt{P^{2}+\phi_{0}^{2}-1}}{\phi_{0}-\sqrt{P^{2}+\phi_{0}^{2}-1}}\right)+\frac{2 \sqrt{2} \log \left(\frac{-\alpha \phi_{0}+\sqrt{\alpha+2} \sqrt{2\left(P^{2}+R_{b}^{2}-1\right)+\alpha\left(R_{b}-\phi_{0}\right)^{2}}+(\alpha+2) R_{b}}{\sqrt{2} \sqrt{(\alpha+2)\left(P^{2}+\phi_{0}^{2}-1\right)}+2 \phi_{0}}\right)}{\sqrt{\alpha+2}} .
$$

It is straightforward to obtain an expression for the times for any value of $\alpha, \phi_{0}$ and for large $R_{b}$

$$
\begin{aligned}
t= & \log \left(\frac{\left(\phi_{0}-1\right)\left(\mu+P \phi_{0}\right)}{\left(\phi_{0}+1\right)\left(\mu-P \phi_{0}\right)}\right)+2 \operatorname{arccoth}\left(\phi_{0}\right) \\
& +2 \sqrt{\frac{2}{\nu}} \operatorname{arccoth}\left(\frac{\sqrt{\alpha+2} P}{\sqrt{\nu}}\right)-\sqrt{\frac{2}{\nu}} \operatorname{arccoth}\left(\frac{\mu(\alpha+2) P}{\sqrt{2 \nu} \phi_{0}-\nu+(\alpha+2) P^{2}}\right) \\
& -\sqrt{\frac{2}{\nu}} \operatorname{arccoth}\left(\frac{\mu(\alpha+2) P}{\sqrt{2 \nu} \phi_{0}+\nu-(\alpha+2) P^{2}}\right)+O\left(1 / R_{b}^{2}\right),
\end{aligned}
$$

where we have defined $\nu \equiv \alpha\left(1-\phi_{0}^{2}\right)+2, \mu \equiv \sqrt{P^{2}+\phi_{0}^{2}-1}$. It is possible to get analytically the form of $V(t)$, expanding both $V[P]$ and $t[P]$ close to $P= \pm 1$. This yields a surprisingly 


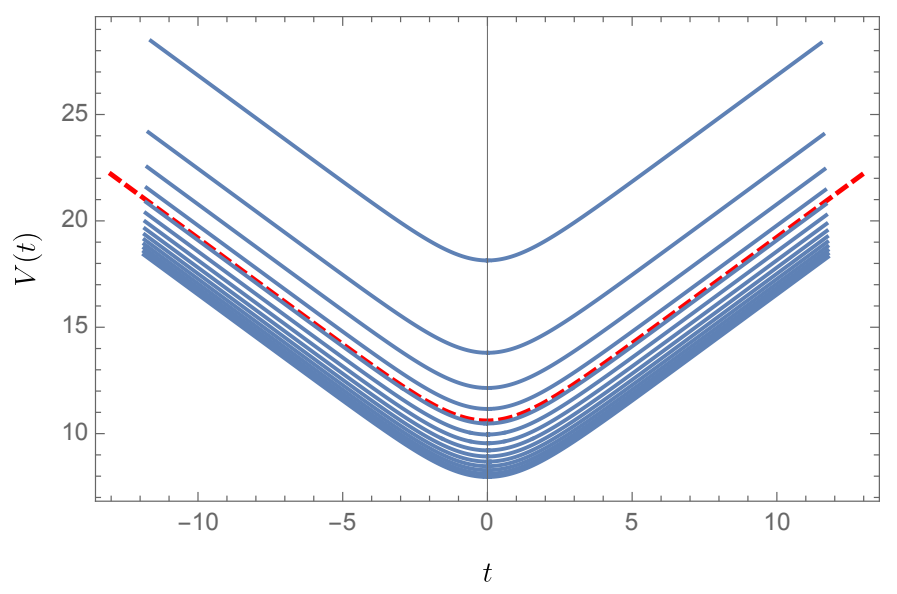

Figure 12. Volume as a function of boundary time $t$ for the different AdS-to-AdS geometries with $R_{b}=100$ and $\phi_{0}=2$. The curves go from $\alpha=-1.9$ at the top to $\alpha=5$ at the bottom, in steps of 0.5. In all cases, for long times, the behaviour is linear with the same slope. In dashed red, we show the curve for the AdS black hole, corresponding to the case $\alpha=0$.

simple expression

$$
V(t)=\frac{2 \sqrt{2} \log R_{b}}{\sqrt{\alpha+2}}+t+\ldots,
$$

where independently of $\alpha$ and $\phi_{0}$, the volume grows linearly in time with coefficient 1 . This can be further seen in figure 12, where we plot the volume for different values of $\alpha$, keeping $\phi_{0}$ fixed. We see that in all cases, the volume grows linearly in time, as in the AdS black hole, which is strikingly different from the cases with dS interiors.

\section{Discussion}

In this paper, we computed the length of spacelike geodesics in different spacetime geometries in two dimensions. These geometries include $\mathrm{AdS}_{2}$ black holes, $\mathrm{dS}_{2}$ space, centaur and $\gamma$-centaur geometries interpolating the two, and flow geometries interpolating two $\mathrm{AdS}_{2}$ spaces with different cosmological constants. With the exception of the $\mathrm{dS}_{2}$ geometry, all the geometries analysed are two-sided, asymptotically AdS geometries with horizons in the interior. We have seen that the nature of the geometry influences significantly the results for lengths and existence of geodesics at different boundary times. For spacetimes containing a black hole event horizon (even when such a horizon is embedded in a flow geometry) the geodesics exist at all times and their lengths grow linearly in time for a long time. The main result of this paper is that in spacetimes containing a cosmological horizon, we observe a very different behaviour. In this case, the geodesics decrease in length for a short time of the order of the inverse temperature ${ }^{23}$ and then they stop existing. These interesting features of cosmological geodesics raise a number of interesting questions regarding their interpretation in terms of complexity of states in the boundary theory and other interesting points which we discuss below.

\footnotetext{
${ }^{23}$ In certain cases this decrease is replaced or preceded by a short period of quadratic increase.
} 
The complexity equals volume conjecture. In the black hole case, spacelike geodesics anchored at the same time on both boundaries always exist. Therefore, the prescription to define complexity as the volume of these geodesics seems reasonable. In some of the geometries analysed in the present paper this is not the case: geodesics anchored at the same time only exist for a short time of the order of the inverse temperature. However, from a boundary perspective, we do not expect that after some time complexity stops being defined. On the contrary, it has been proposed as a measure for late-time entanglement properties. One possibility to solve this apparent contradiction is to consider geodesics that go through future/past infinity which will have infinite length [68]. In this case, we expect the validity of the semi-classical approximation to be lost, but the behaviour of the length is nevertheless intriguing. It decreases for a short time and then jumps to infinity instantaneously. This resonates with the idea of "hyperfast" complexity growth recently explored in [68]. In that paper, the geodesics in pure dS were considered where a temporal boundary is absent. Therefore, the complexity was associated with a notion of time evolution directly on the dS horizon. The interpretation suggested for the complexity becoming infinite after times of the order of the inverse temperature was that of a model whose Hamiltonian couples a significant portion of the system's degrees of freedom within each of its terms. Our approach utilizes a different notion of time evolution and it will be interesting to understand the relation between the two. It is curious to note that if the dual to boundary complexity is instead assumed to be the volume that lies only behind the horizon as in [68], we find linear growth behind the dS horizon as shown in equation (5.13). This is valid only for times of the order of the inverse temperature whereas the black hole volume grows quadratically with time at such early times, as can be checked from expanding equation (4.15).

A different possibility is that complexity=volume is not enough after all, and we need another prescription to compute the complexity of the boundary state. It would be interesting to compare the results obtained for the volume with the ones given by other proposals such as the complexity=action - see [64,70] for computation in JT gravity -, and complexity=spacetime-volume [67]. Those two last proposals are presumably well defined for all boundary times (but possibly also yield divergent answers).

Even though the calculations in the present paper only involve geometries with trivial topology, it is also interesting to compare our results with the recent non-perturbative definition of length proposed in [59]. Under certain assumptions for the potential $U(\phi)$ (see $[71,72]$ ), the authors of [59] find a universal linear growth in the length for times between the thermalization time and $e^{S_{0}}$, independent of the form of the potential. It would be interesting to understand how the arguments of [59] break down in our case.

Shockwaves and out-of-time-ordered correlators. Another interesting observable that probes quantum chaos is the out-of-time-ordered correlator (OTOC). It has been shown that both the exponential growth of the OTOC and the linear growth of complexity are related to the chaotic nature of the system under consideration [54, 73]. As mentioned, the OTOC in an interpolating geometry with a dS horizon does not exhibit exponential growth [35]. Another evidence for the unusual behaviour of the system is found in the 
results of the present paper where the length does not grow linearly with time for long times. Close to the boundary, these geometries have a Schwarzian-like behaviour, governed by the following action [35, 74-76],

$$
S_{\mathrm{bdy}}=\frac{\phi_{b}}{8 \pi G_{N}} \int d u\left(\frac{\gamma}{2}\left(\partial_{u} \tau(u)\right)^{2}-\operatorname{Sch}[\tau(u), u]\right),
$$

which might suggest that the OTOC behaves like $\sim \cos \sqrt{\gamma} t$, giving exponential growth for negative $\gamma$ geometries. However, the results obtained in this paper show that there is no linear growth for the geodesic length even in that case. This is due to the fact that the horizon interior is filled with $\mathrm{dS}$ spacetime. If a precise relation between complexity and the OTOC is established this would suggest that interesting cancellations should happen in the OTOC between the boundary Schwarzian action and the interior modes. It would be interesting to confirm this fact by either doing a direct calculation of the OTOC following [35] or by computing the geodesic length in a shockwave setup. ${ }^{24}$

The volume in shockwave geometries was related to the complexity growth of the precursor operator encoding the influence of a perturbation inserted some time $t_{w}$ in the past on the system $[54,57,58]$. In terms of $t_{w}$, the complexity grows initially exponentially according to the Lyapunov exponent of maximally chaotic systems $\lambda_{L}=2 \pi T$ [82] and then at the scrambling time $t_{*}=(1 / 2 \pi T) \log S$ [73], it starts growing linearly at a rate which is twice the usual linear growth of complexity in the black hole background. It would be useful to study this observable in the centaur geometries to characterize chaos and scrambling in those systems.

Complexity of formation. The length of our geodesic is regulated by the finite location of the boundary. This has a large effect on the complexity, but this large effect does not depend on time. It was suggested in [83] that a useful way to subtract the effect of the boundary, which functions here as a UV regulator, is to consider differences between the complexity of our system and a reference system which was taken to be the AdS vacuum. This vacuum-subtracted complexity goes under the name of complexity of formation

$$
\Delta \mathcal{C}=\mathcal{C}-\mathcal{C}_{\text {global }}=\frac{\Phi_{0}}{G_{N} \ell}\left(V-V_{\text {global }}\right)
$$

where, as opposed to the higher dimensional case [83], here we subtract a single copy of $\mathrm{AdS}_{2}$ since global $\mathrm{AdS}_{2}$ geometry has two boundaries. Evaluating this for the $\mathrm{AdS}_{2}$ black hole, we find using equation (4.15)

$$
\Delta \mathcal{C}_{B H}(t)=8 S_{0} \log (\cosh (\pi T t))+O\left(1 / R_{b}\right)
$$

which simply vanishes for $t=0$ because it is essentially the same space. For the centaur, we find using equation (5.9)

$$
\Delta \mathcal{C}_{\text {centaur }}(t)=\pi+8 S_{0} \log (\cos (\pi T t))+O\left(1 / R_{b}\right), \quad|t| T<1 / 2 .
$$

\footnotetext{
${ }^{24}$ Shockwaves in the centaur geometry were considered in [35]. Other studies of shockwaves in dS space include [77-81].
} 
At $t=0$, this gives $\pi$, which is exactly the length in the $\mathrm{dS}_{2}$ part as we could have expected. Similarly to the higher dimensional case, also here the difference in complexities (at $t \neq 0$ ) grows linearly with the thermal entropy. It is curious to note that the two formulas are related by analytic continuation, but only for short times. Although the volume is always positive, note that the expression (6.4) can become negative. This contrasts with the theorem proven in [84] for asymptotically AdS spacetimes in four dimensions. It would be interesting to use our example to find limitations on possible generalizations of this theorem to different dimensions.

Towards a microscopic quantum theory for dS. If the volume is connected to some notion of complexity, then it would be interesting to see what is needed from the boundary perspective to obtain this type of complexity. We have already a lot of evidence showing that the cosmological horizon is not a quantum maximally chaotic horizon like the black hole one. Given we are studying the problem in two dimensions, the dual theory will be quantum mechanics and we might be able to compute complicated quantities such as the complexity or the OTOC in microscopic models or even try to reverse-engineer models to behave in accordance with our holographic results, possibly along the line of [85] or the SYK RG-flows of [38].

Complex geodesics and dS 2-pt function. Finally, it is interesting to discuss the geodesics in pure $\mathrm{dS}_{2}$. In particular, the length of the geodesics should be related to the twopoint function of heavy fields in $\mathrm{dS}_{2}$ through a saddle-point approximation. The two-point function is known for scalar fields of arbitrary mass at any two points [2]. In particular, at any finite time, it is non-vanishing for points at opposite sides of the circle, as the ones we analysed in this work. However, we showed that there are no real, finite, geodesics anchored at any time different than $t=0$. This apparent contradiction might be solved by the inclusion of complex saddles for the geodesics. The relevance of these saddles in holography remain an interesting open question. We hope to return to this in future work.

\section{Acknowledgments}

We gratefully acknowledge discussions with Dionysios Anninos, Dimitrios Giataganas, Luca Iliesiu and David Vegh. The work of SC is supported by the Israel Science Foundation (grant No. 1417/21) and by the German Research Foundation through a German-Israeli Project Cooperation (DIP) grant "Holography and the Swampland". SC acknowledges the support of Carole and Marcus Weinstein through the BGU Presidential Faculty Recruitment Fund. The work of DAG is funded by the Royal Society under the grant "The Resonances of a de Sitter Universe" and the ERC Consolidator Grant N. 681908, "Quantum black holes: A microscopic window into the microstructure of gravity". EDK is supported by the Israel Science Foundation (grant No. 1111/17) awarded to Eric Kuflik. 


\section{A Thermodynamics of dilaton-gravity theories and $\gamma$-centaur geometries}

In this appendix, we study the thermodynamics of dilaton-gravity theories with a general dilaton potential. This has been studied previously in a variety of different contexts, including [34, 86-88]. For any continuous dilaton potential, the temperature, entropy and specific heat are given by

$$
T=\frac{U\left(\phi\left(r_{h}\right)\right)}{4 \pi}, \quad S=\frac{\Phi_{0}+\phi\left(r_{h}\right)}{4 G_{N}}, \quad C=\frac{1}{4 G_{N}} \frac{U\left(\phi\left(r_{h}\right)\right)}{\partial_{\phi} U\left(\phi\left(r_{h}\right)\right)},
$$

where we have set $\ell=1 .^{25}$ The thermal partition function and energy are given by

$$
\log Z=S-\frac{E}{T}, \quad E=-\frac{1}{16 \pi G_{N}} \int_{r_{h}}^{R_{b}} d \tilde{r} U(\phi(\tilde{r})),
$$

where $R_{b}$ is the location of the Euclidean $\mathrm{AdS}_{2}$ boundary. The energy diverges in the limit $R_{b} \rightarrow \infty$. This divergence can be absorbed in an infinite shift of the ground state energy. It is more meaningful to consider the energy difference between two solutions

$$
\Delta E=E_{r_{h}}-E_{r_{h}^{\prime}}=\frac{1}{16 \pi G_{N}} \int_{r_{h}^{\prime}}^{r_{h}} d \tilde{r} U(\phi(\tilde{r})) .
$$

We obtain the thermodynamics for the interpolating geometries studied in the main text by choosing the appropriate dilaton potentials. Let us consider $U(\phi)=2\left(\left|\phi-\phi_{0}\right|-\phi_{0}\right)$, see figure 13 .

For fixed $\phi_{0}$, there are at most two values of $r_{h}$ corresponding to any given temperature. The metric (2.4) obtained with the choice $r_{h}>\phi_{0}$, will have $R=-2$ everywhere. If in addition we have $\phi_{0}>0$, we have solutions for all $T \geq 0$. On the other hand, for $\phi_{0}<0$, our solutions will always have $T \geq T_{\min }=\left|\phi_{0}\right| / 2 \pi$, so there are no zero-temperature solutions in this case. The specific heat of all solutions with $r_{h}>\phi_{0}$ is given by $C(T)=\pi T / 2 G_{N}$.

To have an interpolating solution such as the ones analysed in the main text, we need $r_{h}<\phi_{0}$. Using eq. (2.4), we can show that in this case, the metric is given by ${ }^{26}$

$$
f(r)_{\gamma}= \begin{cases}r_{h}^{2}-r^{2}, & -\infty<r<\phi_{0}, \\ r_{h}^{2}+r^{2}+2 \phi_{0}\left(\phi_{0}-2 r\right), & \phi_{0}<r<\infty .\end{cases}
$$

When $\phi_{0}>0$, we have to make sure that $r_{h}$ is chosen such that the metric is everywhere positive, see right panel of figure 13. This restricts $r_{h}<-\sqrt{2} \phi_{0}$. For $\phi_{0}<0$, the metric will be everywhere positive, but we still have to make sure that $r_{h}<\phi_{0}$. Combining these two conditions, we find $r_{h}<\phi_{0}<\frac{1}{\sqrt{2}}\left|r_{h}\right|$ which upon setting $r_{h}=-1$ restores the range of $\phi_{0}$ in the main text. In all interpolating solutions, the temperature is given by

$$
T=\frac{\left|r_{h}-\phi_{0}\right|-\phi_{0}}{2 \pi}=\frac{\left|r_{h}\right|}{2 \pi} .
$$

Note that from the above constraints it follows that for $\phi_{0}<0$, there only exist interpolating solutions with $T>\left|\phi_{0}\right| / 2 \pi$ while for $\phi_{0}>0$ solutions exist only for $T>\phi_{0} / \sqrt{2} \pi$.

\footnotetext{
${ }^{25}$ In this appendix we introduce back the factors of $r_{h}$. This is important for computing the thermodynamic quantities.

${ }^{26}$ Note, that the $\phi_{0}$ used here is $\left|r_{h}\right|$ times the $\phi_{0}$ used in the main text. We keep using $\ell=1$.
} 

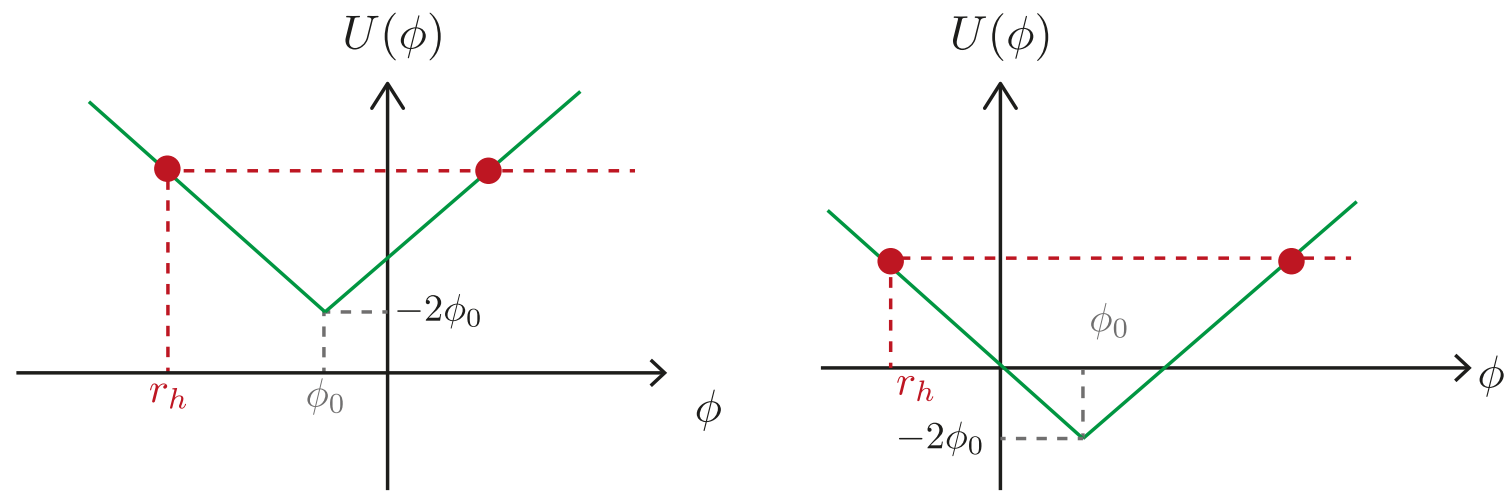

Figure 13. The $\gamma$-centaur potential for $\phi_{0}<0$ (left) and $\phi_{0}>0$ (right). The region where the slope is negative describes $\mathrm{dS}$ while the region where the slope is positive describes AdS and the crossing between the two regions happens at $\phi=\phi_{0}$. There are at most two different values of $r_{h}$ corresponding to a given temperature $T=U\left(\phi\left(r_{h}\right)\right) / 4 \pi$ indicated by red dots in this illustration. To have interpolating solutions we need $r_{h}<\phi_{0}$.

It is straightforward to compute the different thermodynamic quantities for the interpolating geometries. The entropy and the specific heat are given by

$$
S(T)=\frac{\Phi_{0}-2 \pi T}{4 G_{N}} \quad, \quad C(T)=-\frac{\pi T}{2 G_{N}},
$$

where it should be noted that the minus sign comes from having $r_{h}<0$. There are also solutions with positive specific heat, but these have a dilaton that decreases towards the boundary, see $[34,35]$. An important feature of these geometries is that the specific heat is linear in the temperature. The energy is given by,

$$
E(T)=-\frac{1}{16 \pi G_{N}}\left(R_{b}^{2}-4 \phi_{0} R_{b}+2 \phi_{0}^{2}+4 \pi^{2} T^{2}\right),
$$

which as mentioned earlier diverges as $R_{b} \rightarrow \infty$. Nevertheless, only the last term depends on the temperature. Note, that this term is finite and does not depend on $\phi_{0}$. The regulated energy is $\Delta E=-\frac{\pi}{4 G_{N}} \Delta\left(T^{2}\right)$. Note that apart from the ground state energy, thermodynamic quantities do not depend on $\phi_{0}$.

\section{B From conformal coordinates to the Schwarzschild gauge}

In the main text we use mostly Schwarzschild coordinates for the metric. This is a useful gauge to compute the length of geodesics. For the $\gamma$-centaur geometries, though, it is sometimes useful to go to the conformal gauge. In this appendix, we show how to go between the two coordinate systems. We start with the metric that was used in the AdS portion of the $\gamma$-centaur geometries in section 5.2:

$$
d s_{\gamma}^{2}=-f(r)_{\gamma} d t^{2}+\frac{d r^{2}}{f(r)_{\gamma}} \quad, \quad f(r)_{\gamma}=1+r^{2}+2 \phi_{0}\left(\phi_{0}-2 r\right)
$$

Note that this can be written as

$$
f(r)_{\gamma}=\left(r-r_{+}\right)\left(r+r_{-}\right) \quad \text { with } \quad r_{ \pm}=2 \phi_{0} \pm \sqrt{-1+2 \phi_{0}^{2}} .
$$


We can define $\tilde{r}=r-2 \phi_{0}$. In terms of this coordinate, $f(\tilde{r})=\tilde{r}^{2}+\left(1-2 \phi_{0}^{2}\right)$. We want to compare this metric to the one in conformal coordinates. The general negative curvature solution is given by

$$
d s^{2}=\frac{\gamma}{\sin ^{2} \sqrt{\gamma} \rho}\left(-d t^{2}+d \rho^{2}\right)
$$

We can always rescale $\rho$ and $t$ and (B.3) will locally look like the $\mathrm{AdS}_{2}$ black hole metric. However, to fix the temperature of the flow geometries, we fix the periodicity of the time coordinate (in Euclidean signature) using the dS metric, so, globally, all these $\gamma$-centaur geometries are different [35].

It is now straightforward to perform the change of coordinates from Schwarzschild to conformal gauge by identifying

$$
\tilde{r}=\sqrt{\gamma\left(\csc ^{2}(\sqrt{\gamma} \rho)-1\right)}, \text { with } \gamma \equiv 1-2 \phi_{0}^{2},
$$

where you might identify the last parameter as it has been extensively used across section 5.2.

\section{Exact volume and boundary time in $\gamma$-centaur geometries}

In this appendix, we collect several exact results regarding the volume and boundary times in $\gamma$-centaur geometries in section 5.2. We work with the blackening factor (5.15), which has a dS region and an AdS region. As can be observed, the metric in the dS part, is the same as in previous examples. Therefore, the turning point is also at the same location

$$
r_{t}=-\sqrt{1+P^{2}} \text {. }
$$

The tortoise coordinate will change slightly, as the interpolation curve changes from $r=0$ to $r=\phi_{0}$. We will write the formulas for the $\phi_{0}>0$ case, though they can be extended for $\phi_{0}<0$. The tortoise coordinate satisfying $r^{*}(r \rightarrow \infty)=0$ is given by

$$
r^{*}(r)_{\gamma}= \begin{cases}\frac{1}{2} \log \left|\frac{r+1}{r-1}\right|+c_{2}, & -\infty<r<\phi_{0}, \\ \frac{1}{\sqrt{1-2 \phi_{0}^{2}}}\left(\arctan \left(\frac{r-2 \phi_{0}}{\sqrt{1-2 \phi_{0}^{2}}}\right)-\pi / 2\right), & \phi_{0}<r<\infty,\end{cases}
$$

where the constant $c_{2}$ is chosen so that the coordinate is continuous along $\phi_{0}$

$$
c_{2}=-\operatorname{arctanh} \phi_{0}-\frac{1}{\sqrt{1-2 \phi_{0}^{2}}}\left(\arctan \left(\frac{\phi_{0}}{\sqrt{1-2 \phi_{0}^{2}}}\right)+\pi / 2\right) .
$$

Evaluating it at the turning point, we obtain,

$$
r_{t}^{*}=-\operatorname{arctanh} \phi_{0}-\frac{1}{\sqrt{1-2 \phi_{0}^{2}}}\left(\arctan \left(\frac{\phi_{0}}{\sqrt{1-2 \phi_{0}^{2}}}\right)+\pi / 2\right)-\operatorname{arcsinh}\left(\frac{1}{|P|}\right) .
$$


Note how $\gamma$ naturally appears in the expressions. Using the above, it is possible to evaluate the volume and boundary time as a function of the momentum $P$. The volume as a function of $P$ reads

$$
\begin{aligned}
V[P] & =\pi+2 \arctan \left(\frac{\phi_{0}}{\sqrt{P^{2}-\phi_{0}^{2}+1}}\right)+2 \log \left(\frac{R_{b}-2 \phi_{0}+\sqrt{1+P^{2}+R_{b}^{2}-4 \phi_{0} R_{b}+2 \phi_{0}^{2}}}{\sqrt{P^{2}-\phi_{0}^{2}+1}-\phi_{0}}\right) \\
& =\pi+2 \log 2 R_{b}+2 \arctan \left(\frac{\phi_{0}}{\sqrt{1+P^{2}-\phi_{0}^{2}}}\right)-2 \log \left(\sqrt{1+P^{2}-\phi_{0}^{2}}-\phi_{0}\right)+O\left(1 / R_{b}\right)
\end{aligned}
$$

The expression for the time as a function of $P$ is more complicated. For $\phi_{0}>-1 / \sqrt{2}$, we obtain $^{27}$

$$
t \equiv t_{L}+t_{R}=\log \left(\frac{\sqrt{P^{2}-\phi_{0}^{2}+1}-P \phi_{0}}{\sqrt{P^{2}-\phi_{0}^{2}+1}+P \phi_{0}}\right)+\frac{\arctan _{2}\left(x_{1}, y_{1}\right)+\arctan _{2}\left(x_{2}, y_{2}\right)}{\sqrt{1-2 \phi_{0}^{2}}}
$$

where the function $\arctan _{2}(x, y)$ gives the arctangent of $y / x$, taking into account which quadrant the point $(x, y)$ is in and

$$
\begin{aligned}
& x_{1} \equiv-3\left(P^{2}+1\right) \phi_{0}^{2}+P^{2}+2 \phi_{0}^{4}+1 \\
& y_{1} \equiv-2 P \phi_{0} \sqrt{1-2 \phi_{0}^{2}} \sqrt{P^{2}-\phi_{0}^{2}+1} \\
& x_{2} \equiv-P^{2}\left(-4 \phi_{0} R_{b}+R_{b}^{2}+6 \phi_{0}^{2}-1\right)-\left(2 \phi_{0}^{2}-1\right)\left(-4 \phi_{0} R_{b}+R_{b}^{2}+2 \phi_{0}^{2}+1\right), \\
& y_{2} \equiv 2 P \sqrt{1-2 \phi_{0}^{2}}\left(2 \phi_{0}-R_{b}\right) \sqrt{-4 \phi_{0} R_{b}+R_{b}^{2}+P^{2}+2 \phi_{0}^{2}+1}
\end{aligned}
$$

Plotting the boundary time for different possible values of $\phi_{0}$ allows us to see different interesting patterns for the geodesics. For $\left|\phi_{0}\right|<\frac{1}{\sqrt{2}}$, each time has at most one specific value of $P$, and therefore one geodesic, associated with it, see figure $14 \mathrm{a}$. The geodesics stop existing at a time of the order of the inverse temperature, given by equation (5.17). For $-1<\phi_{0}<$ $-\frac{1}{\sqrt{2}}$, the situation is more interesting. In that range, at very early times, there are three different maximal geodesics anchored at the very same boundary time. Depending on the value of $\phi_{0}$, there is another range of times where there are two geodesics at the same time — see figure $14 \mathrm{c}$ - or only one - see figure 14b. At larger times, there are no geodesics.

\footnotetext{
${ }^{27}$ For $\phi_{0}<-1 / \sqrt{2}$, part of the expression becomes complex. In order to obtain the right value for the boundary times, one has to take the real part of this expression.
} 


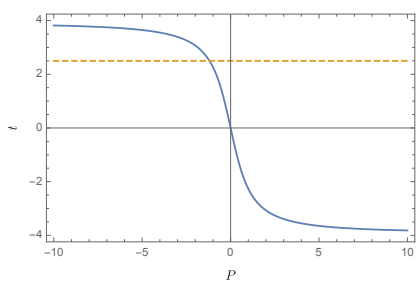

(a) $\phi_{0}=0.2$

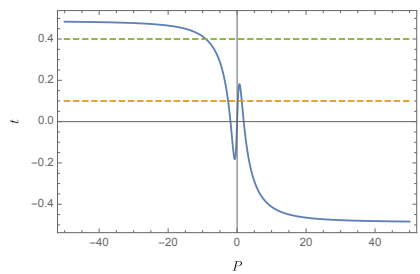

(b) $\phi_{0}=-0.85$

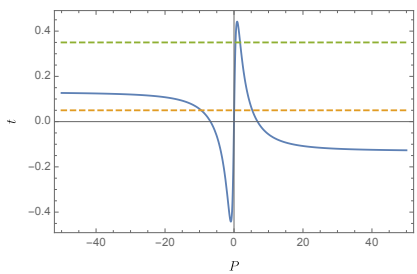

(c) $\phi_{0}=-0.95$

Figure 14. $t$ as a function of $P$ for different values of $\phi_{0}$. In each figure, the colours of the dashed lines correspond to those of the relevant geodesics in figure 8. $R_{b}$ is set to 10 . In the first plot, each time corresponds to at most a single value of $P$. In the second plot, at short times there are three relevant values of $P$ (yellow dashed line), while at later times, there is only one relevant value of $P$ (green dashed line). In the last plot, the yellow dashed line shows again three relevant values of $P$, but the green line intersects at two different values of $P$.

Open Access. This article is distributed under the terms of the Creative Commons Attribution License (CC-BY 4.0), which permits any use, distribution and reproduction in any medium, provided the original author(s) and source are credited.

\section{References}

[1] D. Anninos, de Sitter Musings, Int. J. Mod. Phys. A 27 (2012) 1230013 [arXiv:1205.3855] [INSPIRE].

[2] M. Spradlin, A. Strominger and A. Volovich, Les Houches lectures on de Sitter space, in Les Houches Summer School: Session 76: Euro Summer School on Unity of Fundamental Physics: Gravity, Gauge Theory and Strings, pp. 423-453 (2001) [hep-th/0110007] [INSPIRE].

[3] G.W. Gibbons and S.W. Hawking, Cosmological Event Horizons, Thermodynamics, and Particle Creation, Phys. Rev. D 15 (1977) 2738 [InSPIRE].

[4] O. Aharony, S.S. Gubser, J.M. Maldacena, H. Ooguri and Y. Oz, Large N field theories, string theory and gravity, Phys. Rept. 323 (2000) 183 [hep-th/9905111] [INSPIRE].

[5] D. Harlow, Jerusalem lectures on black holes and quantum information, Rev. Mod. Phys. 88 (2016) 015002.

[6] M. Rangamani and T. Takayanagi, Holographic Entanglement Entropy, Lecture Notes in Physics, Springer International Publishing (2017) [DOI] [arXiv: 1609.01287] [INSPIRE].

[7] L. Susskind, Three Lectures on Complexity and Black Holes, SpringerBriefs in Physics, Springer (2018) [DOI] [arXiv:1810.11563] [INSPIRE].

[8] A. Strominger, The dS/CFT correspondence, JHEP 10 (2001) 034 [hep-th/0106113] [INSPIRE].

[9] E. Witten, Quantum gravity in de Sitter space, in Strings 2001: International Conference, (2001) [hep-th/0106109] [INSPIRE].

[10] A. Strominger, Inflation and the dS/CFT correspondence, JHEP 11 (2001) 049 [hep-th/0110087] [INSPIRE]. 
[11] J.M. Maldacena, Non-Gaussian features of primordial fluctuations in single field inflationary models, JHEP 05 (2003) 013 [astro-ph/0210603] [INSPIRE].

[12] D. Anninos, T. Hartman and A. Strominger, Higher Spin Realization of the $d S / C F T$ Correspondence, Class. Quant. Grav. 34 (2017) 015009 [arXiv:1108.5735] [InSPIRE].

[13] D. Anninos, F. Denef, R. Monten and Z. Sun, Higher Spin de Sitter Hilbert Space, JHEP 10 (2019) 071 [arXiv: 1711.10037] [InSPIRE].

[14] T. Banks, Some thoughts on the quantum theory of stable de Sitter space, hep-th/0503066 [INSPIRE].

[15] T. Banks, B. Fiol and A. Morisse, Towards a quantum theory of de Sitter space, JHEP 12 (2006) 004 [hep-th/0609062] [INSPIRE].

[16] T. Banks, Holographic spacetime, Int. J. Mod. Phys. D 21 (2012) 1241004 [InSPIRE].

[17] D. Anninos, S.A. Hartnoll and D.M. Hofman, Static Patch Solipsism: Conformal Symmetry of the de Sitter Worldline, Class. Quant. Grav. 29 (2012) 075002 [arXiv:1109.4942] [INSPIRE].

[18] S. Leuven, E. Verlinde and M. Visser, Towards non-AdS Holography via the Long String Phenomenon, JHEP 06 (2018) 097 [arXiv: 1801. 02589] [INSPIRE].

[19] V. Gorbenko, E. Silverstein and G. Torroba, $d S / d S$ and TT,$J H E P 03$ (2019) 085 [arXiv: 1811.07965] [INSPIRE].

[20] A. Lewkowycz, J. Liu, E. Silverstein and G. Torroba, $T \bar{T}$ and EE, with implications for (A)dS subregion encodings, JHEP 04 (2020) 152 [arXiv:1909.13808] [INSPIRE].

[21] V. Shyam, $T \bar{T}+\Lambda_{2}$ Deformed CFT on the Stretched $d S_{3}$ Horizon, arXiv:2106.10227 [INSPIRE].

[22] N. Arkani-Hamed, D. Baumann, H. Lee and G.L. Pimentel, The Cosmological Bootstrap: Inflationary Correlators from Symmetries and Singularities, JHEP 04 (2020) 105 [arXiv: 1811.00024] [INSPIRE].

[23] C. Sleight, A Mellin Space Approach to Cosmological Correlators, JHEP 01 (2020) 090 [arXiv: 1906.12302] [INSPIRE].

[24] C. Sleight and M. Taronna, Bootstrapping Inflationary Correlators in Mellin Space, JHEP 02 (2020) 098 [arXiv: 1907.01143] [INSPIRE].

[25] C. Sleight and M. Taronna, From AdS to dS Exchanges: Spectral Representation, Mellin Amplitudes and Crossing, arXiv:2007.09993 [INSPIRE].

[26] M. Hogervorst, J. Penedones and K.S. Vaziri, Towards the non-perturbative cosmological bootstrap, arXiv:2107.13871 [INSPIRE].

[27] L. Di Pietro, V. Gorbenko and S. Komatsu, Analyticity and Unitarity for Cosmological Correlators, arXiv:2108.01695 [INSPIRE].

[28] C. Sleight and M. Taronna, From dS to AdS and back, JHEP 12 (2021) 074 [arXiv:2109.02725] [INSPIRE].

[29] D. Anninos, T. Bautista and B. Mühlmann, The two-sphere partition function in two-dimensional quantum gravity, JHEP 09 (2021) 116 [arXiv:2106.01665] [INSPIRE].

[30] B. Mühlmann, The two-sphere partition function in two-dimensional quantum gravity at fixed area, JHEP 09 (2021) 189 [arXiv:2106.04532] [INSPIRE]. 
[31] R. Mahajan, D. Stanford and C. Yan, Sphere and disk partition functions in Liouville and in matrix integrals, arXiv:2107.01172 [INSPIRE].

[32] S. Kachru, R. Kallosh, A.D. Linde and S.P. Trivedi, de Sitter vacua in string theory, Phys. Rev. D 68 (2003) 046005 [hep-th/0301240] [INSPIRE].

[33] G. Obied, H. Ooguri, L. Spodyneiko and C. Vafa, de Sitter Space and the Swampland, arXiv: 1806.08362 [INSPIRE].

[34] D. Anninos and D.M. Hofman, Infrared Realization of $d S_{2}$ in AdS $S_{2}$, Class. Quant. Grav. 35 (2018) 085003 [arXiv: 1703.04622] [INSPIRE].

[35] D. Anninos, D.A. Galante and D.M. Hofman, de Sitter horizons 83 holographic liquids, JHEP 07 (2019) 038 [arXiv: 1811.08153] [inSPIRE].

[36] B. Freivogel, V.E. Hubeny, A. Maloney, R.C. Myers, M. Rangamani and S. Shenker, Inflation in AdS/CFT, JHEP 03 (2006) 007 [hep-th/0510046] [INSPIRE].

[37] D.A. Lowe and S. Roy, Punctuated eternal inflation via AdS/CFT, Phys. Rev. D 82 (2010) 063508 [arXiv: 1004.1402] [inSPIRE].

[38] D. Anninos and D.A. Galante, Constructing AdS $S_{2}$ flow geometries, JHEP 02 (2021) 045 [arXiv: 2011.01944] [INSPIRE].

[39] A. Almheiri and J. Polchinski, Models of AdS 2 backreaction and holography, JHEP 11 (2015) 014 [arXiv: 1402.6334] [INSPIRE].

[40] K. Jensen, Chaos in AdS 2 Holography, Phys. Rev. Lett. 117 (2016) 111601 [arXiv: 1605.06098] [INSPIRE].

[41] J. Maldacena, D. Stanford and Z. Yang, Conformal symmetry and its breaking in two dimensional Nearly Anti-de-Sitter space, PTEP 2016 (2016) 12C104 [arXiv:1606.01857] [INSPIRE].

[42] J. Engelsöy, T.G. Mertens and H. Verlinde, An investigation of $A d S_{2}$ backreaction and holography, JHEP 07 (2016) 139 [arXiv: 1606.03438] [INSPIRE].

[43] J. Maldacena and D. Stanford, Remarks on the Sachdev-Ye-Kitaev model, Phys. Rev. D 94 (2016) 106002 [arXiv: 1604.07818] [INSPIRE].

[44] G. Sárosi, $A d S_{2}$ holography and the SYK model, PoS Modave2017 (2018) 001 [arXiv:1711.08482] [INSPIRE].

[45] S. Gao and R.M. Wald, Theorems on gravitational time delay and related issues, Class. Quant. Grav. 17 (2000) 4999 [gr-qc/0007021] [INSPIRE].

[46] D. Anninos, F. Denef, Y.T.A. Law and Z. Sun, Quantum de Sitter horizon entropy from quasicanonical bulk, edge, sphere and topological string partition functions, JHEP 01 (2022) 088 [arXiv: 2009. 12464] [INSPIRE].

[47] D. Anninos and E. Harris, Three-dimensional de Sitter horizon thermodynamics, JHEP 10 (2021) 091 [arXiv:2106.13832] [INSPIRE].

[48] J.L.F. Barbón and E. Rabinovici, Holographic complexity and spacetime singularities, JHEP 01 (2016) 084 [arXiv: 1509.09291] [INSPIRE].

[49] A. Reynolds and S.F. Ross, Complexity in de Sitter Space, Class. Quant. Grav. 34 (2017) 175013 [arXiv: 1706.03788] [INSPIRE]. 
[50] H. Geng, Some Information Theoretic Aspects of De-Sitter Holography, JHEP 02 (2020) 005 [arXiv: 1911.02644] [INSPIRE].

[51] J. Watrous, Quantum Computational Complexity, in Encyclopedia of Complexity and Systems Science, R. Meyers ed., Springer (2009) [DOI].

[52] S. Aaronson, The Complexity of Quantum States and Transformations: From Quantum Money to Black Holes, arXiv:1607.05256 [INSPIRE].

[53] L. Susskind, Computational Complexity and Black Hole Horizons, Fortsch. Phys. 64 (2016) 24 [Addendum ibid. 64 (2016) 44] [arXiv:1403.5695] [INSPIRE].

[54] D. Stanford and L. Susskind, Complexity and Shock Wave Geometries, Phys. Rev. D 90 (2014) 126007 [arXiv: 1406 .2678] [INSPIRE].

[55] L. Susskind, Entanglement is not enough, Fortsch. Phys. 64 (2016) 49 [arXiv:1411.0690] [INSPIRE].

[56] D. Carmi, S. Chapman, H. Marrochio, R.C. Myers and S. Sugishita, On the Time Dependence of Holographic Complexity, JHEP 11 (2017) 188 [arXiv:1709.10184] [INSPIRE].

[57] S. Chapman, H. Marrochio and R.C. Myers, Holographic complexity in Vaidya spacetimes. Part I, JHEP 06 (2018) 046 [arXiv: 1804.07410] [INSPIRE].

[58] S. Chapman, H. Marrochio and R.C. Myers, Holographic complexity in Vaidya spacetimes. Part II, JHEP 06 (2018) 114 [arXiv: 1805.07262] [INSPIRE].

[59] L.V. Iliesiu, M. Mezei and G. Sárosi, The volume of the black hole interior at late times, arXiv:2107.06286 [INSPIRE].

[60] J.F. Pedraza, A. Russo, A. Svesko and Z. Weller-Davies, Lorentzian Threads as Gatelines and Holographic Complexity, Phys. Rev. Lett. 127 (2021) 271602 [arXiv:2105.12735] [InSPIRE].

[61] J.F. Pedraza, A. Russo, A. Svesko and Z. Weller-Davies, Sewing spacetime with Lorentzian threads: complexity and the emergence of time in quantum gravity, JHEP 02 (2022) 093 [arXiv:2106.12585] [INSPIRE].

[62] S. Ryu and T. Takayanagi, Holographic derivation of entanglement entropy from AdS/CFT, Phys. Rev. Lett. 96 (2006) 181602 [hep-th/0603001] [INSPIRE].

[63] M. Freedman and M. Headrick, Bit threads and holographic entanglement, Commun. Math. Phys. 352 (2017) 407 [arXiv: 1604.00354] [INSPIRE].

[64] A.R. Brown, H. Gharibyan, H.W. Lin, L. Susskind, L. Thorlacius and Y. Zhao, Complexity of Jackiw-Teitelboim gravity, Phys. Rev. D 99 (2019) 046016 [arXiv:1810.08741] [INSPIRE].

[65] A.R. Brown, D.A. Roberts, L. Susskind, B. Swingle and Y. Zhao, Complexity, action, and black holes, Phys. Rev. D 93 (2016) 086006 [arXiv:1512.04993] [InSPIRE].

[66] A.R. Brown, D.A. Roberts, L. Susskind, B. Swingle and Y. Zhao, Holographic Complexity Equals Bulk Action?, Phys. Rev. Lett. 116 (2016) 191301 [arXiv:1509.07876] [INSPIRE].

[67] J. Couch, W. Fischler and P.H. Nguyen, Noether charge, black hole volume, and complexity, JHEP 03 (2017) 119 [arXiv:1610.02038] [INSPIRE].

[68] L. Susskind, Entanglement and Chaos in de Sitter Holography: An SYK Example, arXiv:2109.14104 [INSPIRE].

[69] R.M. Wald, General Relativity, Chicago University Press, Chicago, U.S.A. (1984) [DOI]. 
[70] K. Goto, H. Marrochio, R.C. Myers, L. Queimada and B. Yoshida, Holographic Complexity Equals Which Action?, JHEP 02 (2019) 160 [arXiv:1901.00014] [INSPIRE].

[71] H. Maxfield and G.J. Turiaci, The path integral of 3D gravity near extremality; or, JT gravity with defects as a matrix integral, JHEP 01 (2021) 118 [arXiv:2006.11317] [INSPIRE].

[72] E. Witten, Matrix Models and Deformations of JT Gravity, Proc. Roy. Soc. Lond. A 476 (2020) 20200582 [arXiv: 2006.13414] [INSPIRE].

[73] S.H. Shenker and D. Stanford, Black holes and the butterfly effect, JHEP 03 (2014) 067 [arXiv: 1306.0622] [INSPIRE].

[74] K. Jensen, Scrambling in nearly thermalized states at large central charge, arXiv:1906.05852 [INSPIRE].

[75] J. Yoon, A bound on chaos from stability, JHEP 11 (2021) 097 [arXiv: 1905.08815] [INSPIRE].

[76] P. Nayak, J. Sonner and M. Vielma, Extended Eigenstate Thermalization and the role of FZZT branes in the Schwarzian theory, JHEP 03 (2020) 168 [arXiv:1907.10061] [INSPIRE].

[77] M. Hotta and M. Tanaka, Shock wave geometry with nonvanishing cosmological constant, Class. Quant. Grav. 10 (1993) 307 [INSPIRE].

[78] M. Hotta and M. Tanaka, Gravitational shock waves and quantum fields in the de Sitter space, Phys. Rev. D 47 (1993) 3323 [InSPIRE].

[79] K. Sfetsos, On gravitational shock waves in curved space-times, Nucl. Phys. B 436 (1995) 721 [hep-th/9408169] [INSPIRE].

[80] L. Aalsma and G. Shiu, Chaos and complementarity in de Sitter space, JHEP 05 (2020) 152 [arXiv: 2002.01326] [INSPIRE].

[81] H. Geng, Non-local entanglement and fast scrambling in de-Sitter holography, Annals Phys. 426 (2021) 168402 [arXiv: 2005.00021] [INSPIRE].

[82] J. Maldacena, S.H. Shenker and D. Stanford, A bound on chaos, JHEP 08 (2016) 106 [arXiv: 1503.01409] [INSPIRE].

[83] S. Chapman, H. Marrochio and R.C. Myers, Complexity of Formation in Holography, JHEP 01 (2017) 062 [arXiv:1610.08063] [INSPIRE].

[84] N. Engelhardt and r. Folkestad, General bounds on holographic complexity, JHEP 01 (2022) 040 [arXiv: 2109. 06883] [INSPIRE].

[85] T. Anous and F.M. Haehl, The quantum p-spin glass model: a user manual for holographers, J. Stat. Mech. 2111 (2021) 113101 [arXiv:2106. 03838] [INSPIRE].

[86] M. Cavaglia, Geometrodynamical formulation of two-dimensional dilaton gravity, Phys. Rev. D 59 (1999) 084011 [hep-th/9811059] [INSPIRE].

[87] D. Grumiller and R. McNees, Thermodynamics of black holes in two (and higher) dimensions, JHEP 04 (2007) 074 [hep-th/0703230] [INSPIRE].

[88] E. Witten, Deformations of JT Gravity and Phase Transitions, arXiv:2006.03494 [INSPIRE]. 